\title{
La porta di entrata per la comprensione di un problema: la lettura del testo
}

\section{The Entrance Door to Understand a Mathematical Problem: Reading the Text}

\author{
Silvia Demartini e Silvia Sbaragli \\ Dipartimento formazione e apprendimento - SUPSI di Locarno, Svizzera
}

Sunto / In questo articolo vengono proposte alcune riflessioni legate al ruolo della lettura concepita come prima e inevitabile "porta d'entrata" per comprendere un problema matematico espresso attraverso un testo, con il fine di risolverlo. L'articolo inizia con un'analisi delle diverse fasi della risoluzione di un problema proposte dalla letteratura in didattica della matematica e si concentra poi su che cosa significa leggere un testo per capirlo; successivamente, si illustrano le diverse modalità di lettura in funzione del tipo, del genere di testo e dei suoi scopi. In particolare, vengono mostrati il ruolo cruciale della lettura intensiva per poter comprendere il testo di un problema matematico e le possibili pratiche didattiche che possono aiutare il docente a favorire questo tipo di lettura in classe. Si conclude l'articolo ribadendo l'importanza di "prendersi il tempo": per il docente di proporre un percorso didattico rivolto alla comprensione del testo e per gli allievi di leggere il testo, in modo da interrogarlo e valutarlo in profondità. Ciò per scoprire attraverso l'esperienza come una migliore lettura potenzi l'efficacia nel problem solving.

Parole chiave: matematica; italiano; problem solving; lettura; comprensione.
Abstract / This paper presents some reflections on the role of reading conceived as the first and inevitable "entrance door" to understand a mathematical word problem, with the aim of solving it. We start from an analysis of the different phases required to solve a problem, shown by the research in Mathematics Education, and then we focus on what it means to read and comprehend a text; afterwards, we illustrate the different types of reading depending on the type and the purposes of a text. Particular attention is given to the role of intensive reading in understanding the text of a mathematical problem, and some possible didactic practices are shortly described to help teachers to improve this reading strategy at school. We conclude the article by stressing the importance of "taking the time": time for teachers to propose interactive didactic paths focused on understanding and discussing texts, and time for pupils to read, question and evaluate texts in depth. This practice will lead students to discover through experience how a better reading can have a positive impact on problem solving.

Keywords: Mathematics; Italian Language; Problem Solving; Reading; Understanding.

L'articolo si inserisce all'interno della ricerca Italmatica. Comprendere la matematica a scuola tra lingua comune e linguaggio specialistico (progetto 176339 del Fondo Nazionale Svizzero per la Ricerca Scientifica), ideata e condotta da due centri di competenza' del Dipartimento Formazione e Apprendimento (DFA) della Scuola Universitaria Professionale della Svizzera Italiana (SUPSI). II progetto, il cui svolgimento è previsto su tre anni accademici (2018-2021), è il naturale proseguimento di studi precedenti, che hanno confermato la necessità di indagare la comprensione in am-

1. Si tratta dei Centri competenze Didattica della Matematica (DdM) e Didattica dell'italiano lingua di scolarizzazione (DILS). 
bito matematico con particolare riferimento agli aspetti linguistici. ${ }^{2}$

La letteratura nel campo della didattica della matematica e la nostra pratica di ricerca hanno spesso mostrato come le difficoltà nell'apprendimento e nella mobilitazione delle competenze in tale disciplina non siano unicamente ascrivibili alla matematica stessa, ma siano spesso collegate a difficoltà di comprensione linguistica, specialmente quando si opera in contesti complessi e ampi come quello della risoluzione di problemi. Tra i recenti risultati di ricerca ottenuti in tal senso, citiamo la valutazione didattica della prova standardizzata di matematica somministrata nel maggio 2015 a tutti gli allievi di quinta elementare del Canton Ticino, che mostra come le risposte sbagliate di diversi studenti siano legate a difficoltà nella comprensione del testo dell'item, in particolare a difficoltà di interpretazione linguistica (Sbaragli \& Franchini, 2017; Franchini, Lemmo \& Sbaragli, 2017).

Nel rapporto riferito a tale ricerca (Sbaragli \& Franchini, 2017) si è messo in evidenza come la risoluzione di problemi, scelti in modo da essere creati sulle competenze degli allievi, coinvolga il processo Matematizzare e modellizzare, che rappresenta una competenza chiave per la formazione del pensiero matematico dell'allievo e uno dei quattro processi cognitivi dell'area matematica del Piano di studio della scuola dell'obbligo ticinese (DECS, 2015). Tale processo si riferisce all'attività di organizzazione e analisi di qualsiasi situazione di realtà attraverso strumenti matematici, cioè alla traduzione, riorganizzazione e (ri)costruzione di un problema all'interno del contesto reale nel mondo simbolico della matematica, e viceversa (Jupri \& Drijvers, 2016).

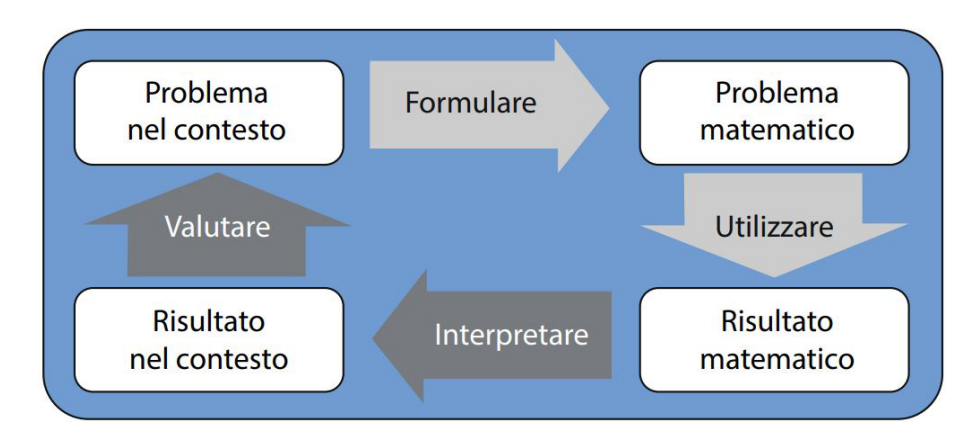

Per quanto concerne la matematizzazione, PISA, Programme for International Student Assessment (OECD, 2004; 2013; 2016), ha messo in evidenza un ciclo, detto appunto ciclo della matematizzazione, che è possibile riassumere nei seguenti aspetti:

- si parte da un problema situato nella realtà ("problema nel contesto");

- si organizza il problema in base a concetti matematici e si identificano gli strumenti matematici pertinenti;

2. Gli esiti delle indagini preliminari sono illustrati in una serie di pubblicazioni: Demartini, Fornara \& Sbaragli (2017; 2018; in corso di stampa), Demartini \& Sbaragli (2015a; 2015b), Fornara \& Sbaragli (2013; 2016; 2017). 
- si eliminano progressivamente gli elementi della realtà attraverso processi quali fare supposizioni, generalizzare e formalizzare, che mettono in evidenza le caratteristiche matematiche della situazione e trasformano il problema reale in uno matematico che rappresenti fedelmente la situazione di partenza (tramite questa fase e la precedente si passa al "problema matematico" grazie al processo "formulare");

- si risolve il problema matematico (si passa al "risultato matematico" grazie al processo "utilizzare");

- si interpreta la soluzione matematica in termini di situazione reale, individuando anche i limiti della soluzione proposta (si passa al "risultato nel contesto" e si ritorna al "problema nel contesto", grazie ai processi "interpretare" e "valutare").

Nella terminologia proposta dall'OECD, tale ciclo è quindi descritto attraverso l'identificazione di alcuni processi che sono ritenuti fondamentali per la gestione del problema: formulare il problema, ovvero trasporlo dal linguaggio naturale al linguaggio formalizzato della disciplina; utilizzare i propri saperi per dare una risposta al problema che si è identificato; interpretare e valutare la pertinenza della soluzione ipotizzata in rapporto al contesto di realtà da cui si è partiti. Per un approfondimento sul tema si veda Franchini, Lemmo \& Sbaragli (2017).

L'esplicitazione di queste fasi permette di analizzare in modo più mirato e consapevole le competenze degli allievi necessarie per risolvere un problema e di prevedere eventuali azioni di intervento specifiche in caso di difficoltà.

In questo articolo ci concentreremo in particolare sul primo processo, formulare, che coinvolge la comprensione del "problema nel contesto" con il fine di trasformarlo in "problema matematico", cioè gestibile attraverso strumenti, concetti e procedure proprie della matematica. Per risolvere un problema è infatti necessario identificare le informazioni rilevanti, depurandole da tutto ciò che è ininfluente alla soluzione, per poi individuare una struttura, un modello astratto e ideale della situazione (ad esempio una formula, un'espressione o equazione algebrica, uno schema) basato sulle ipotesi elaborate, sui concetti e sulle relazioni individuate. Per fare ciò è dunque necessaria una comprensione profonda della situazione, che includa il riconoscimento delle diverse informazioni trasmesse dal testo (anche quelle sottintese) espresse in varie forme (linguistica, aritmetica, algebrica, grafica ecc.). Questo processo coinvolge la comprensione del "problema nel contesto", quindi la comprensione anche linguistica della situazione, con il fine di trasformarlo in "problema matematico": è dunque collegato con la lettura del testo, oggetto di questo articolo.

\section{Il processo formulare a confronto con alcune teorie della risoluzio-
ne di problemi}

L'intento di questo paragrafo è di collegare il primo processo del ciclo della matematizzazione (formulare) con alcune fasi delle più classiche teorie legate alla risoluzione dei problemi (individuate da Polya, 1945 e Schoenfeld, 1983), arrivando così a definire la "porta d'entrata" della risoluzione di un problema: la lettura del testo. 


\subsection{La teoria del problem solving di Polya}

Nel 1945, George Polya, uno dei più grandi studiosi della teoria del problem solving, analizza puntualmente la risoluzione di un problema matematico identificando le quattro seguenti principali fasi nell'azione di risoluzione:

1) la comprensione del problema, per cui è necessario conoscere chiaramente quanto richiesto;

2) la compilazione di un piano che prevede la scoperta dei legami che intercorrono fra le varie informazioni, fra i dati e l'incognita;

3) lo sviluppo di un piano che comporta l'applicazione di regole, algoritmi e procedure;

4) la verifica del risultato, per cui si richiede di esaminare la soluzione ottenuta e di procedere alla verifica e alla discussione.

Le fasi non si susseguono in maniera lineare, ma secondo un ciclo iterativo che si ripete, nel quale il risolutore si muove alla ricerca della soluzione. Inoltre, il passaggio da una fase all'altra non avviene per tutti allo stesso modo: c'è infatti chi si sofferma maggiormente su una fase e chi su un'altra. Va anche aggiunto che tali fasi sono accompagnate da domande chiave che il bravo solutore di problemi si pone in modo spontaneo, così da stimolare le operazioni mentali utili per la risoluzione e per suggerire delle euristiche. Le domande che il bravo risolutore si pone si susseguono con una certa regolarità in corrispondenza di momenti diversi del processo risolutivo. Polya, nel suo progetto didattico, ha cercato di insegnare agli allievi repertori di domande per mettere in gioco le strategie opportune per la risoluzione di un problema. Sull'importanza di porsi domande durante il processo risolutivo dovremo tornare in seguito, tenendo anche conto del fatto che quest'attitudine può essere incentivata e consolidata attraverso mirate attività preparatorie di lettura e lavoro sui testi.

È possibile riscontrare le stesse azioni proposte da Polya all'interno del ciclo della matematizzazione dell'OECD presentato precedentemente, come riportato nel seguente schema.

Tabella 1

Processi del ciclo della

matematizzazione a confronto con le fasi della risoluzione di un problema proposte da Polya.

\begin{tabular}{|l|l|}
\hline Fasi nel ciclo della matematizzazione & $\begin{array}{l}\text { Fasi nella risoluzione di un problema } \\
\text { secondo Polya }\end{array}$ \\
\hline Formulare & $\begin{array}{l}\text { Comprensione del problema } \\
\text { Compilazione di un piano }\end{array}$ \\
\hline Utilizzare & Sviluppo di un piano \\
\hline $\begin{array}{l}\text { Interpretare } \\
\text { Valutare }\end{array}$ & Verifica del risultato \\
\hline
\end{tabular}

\subsection{Gli "episodi” di Schoenfeld}

Per sottolineare meglio l'importanza delle decisioni strategiche all'interno di un comportamento risolutivo, un altro importante ricercatore, Schoenfeld (1983, p. 17), propone di suddividerlo in «frammenti macroscopici di comportamento coerente», detti "episodi", caratterizzati nel modo seguente: 
Tabella 2

Fasi nella risoluzione di un problema secondo Polya

a confronto con gli episodi

nella risoluzione di un

problema secondo

Schoenfeld.
1) Lettura
2) Analisi
3) Esplorazione
4) Pianificazione
5) Implementazione
6) Verifica
7) Transizione

In questi episodi si riconoscono facilmente le quattro fasi di Polya:

\begin{tabular}{|l|l|}
\hline $\begin{array}{l}\text { Fasi nella risoluzione di un problema } \\
\text { secondo Polya }\end{array}$ & $\begin{array}{l}\text { Episodi nella risoluzione di un problema } \\
\text { secondo Schoenfeld }\end{array}$ \\
\hline Comprensione del problema & $\begin{array}{l}\text { Lettura } \\
\text { Analisi } \\
\text { Esplorazione }\end{array}$ \\
\hline Compilazione di un piano & Pianificazione \\
\hline Sviluppo del piano & Implementazione \\
\hline Verifica del risultato & Verifica \\
& Transizione \\
\hline
\end{tabular}

Le differenze maggiori tra le due impostazioni risiedono principalmente nell'individuare nella comprensione del problema tre fasi (episodi): "lettura", "analisi" ed "esplorazione", e nell'introdurre l'episodio "transizione", che consiste in controlli e valutazioni che il soggetto compie durante il processo risolutivo. Va sottolineato come per Schoenfeld la porta d'entrata per la risoluzione di un problema sia la "lettura", che rappresenta la prima e inevitabile fase che l'allievo deve gestire attivando strategie adeguate al contesto.

\subsection{Decisioni strategiche nella risoluzione dei problemi}

Risulta interessante citare anche la ricerca condotta da Schoenfeld nel 1992 - basata sull'osservazione del comportamento di soggetti durante la risoluzione di problemi non standard -, in cui viene messa in evidenza una differenza notevole nella quantità e nella qualità delle decisioni strategiche attivate. Nelle Figure 2 e 3 sono riportati i diagrammi che mostrano tale differenza. A essere particolarmente poco efficaci nei "cattivi solutori" sono la lettura, molto rapida, e la gestione del tempo dedicato a esplorare un unico approccio: nello specifico, i cattivi solutori di problemi (Figura 2) riservano tanto tempo all'esplorazione, dedicandone poco alla comprensione del testo. Un simile comportamento non appare generalmente quando gli studenti affrontano esercizi di routine, dal momento che il contesto in questo caso informa direttamente gli studenti su quali tecniche utilizzare. Per contro, i "bravi solutori" (Figura 3), i solutori esperti, non passano subito all'azione, ma usano parte del tempo per riflettere e per organizzare le informazioni reperite, ponendosi varie domande del tipo Che cosa sto facendo?, Perché lo sto facendo?, Che cosa me ne faccio del risultato? ecc. e decidendo come procedere di conseguenza. Dall'osservazione del grafico (Figura 3) emerge proprio il fatto che il "bravo solutore", in questo caso un 
Figura 2

Grafico della linea

temporale di un cattivo

solutore che risolve un

problema (Schoenfeld,

1992, p. 61)
Figura 3

Grafico della linea

temporale di un bravo

solutore che sta

lavorando a un problema

complesso (Schoenfeld,

1992, p. 62). matematico posto di fronte a un problema difficile, ha speso più della metà del tempo a sua disposizione cercando di dare un senso al problema, dedicandosi cioè alla lettura e all'analisi delle informazioni in esso veicolate. Inoltre, un bravo solutore considera ed esplora con consapevolezza diversi approcci risolutivi possibili, anche quelli sbagliati, passando da un episodio all'altro in funzione della bontà o meno della soluzione trovata. Egli non porta mai fino in fondo gli approcci sbagliati, pur esplorandoli, come fanno invece i cattivi solutori, perché è «tanto inesorabile nel controllare e rifiutare idee quanto ingegnoso nel generarle» (Schoenfeld, 1987, p. 194), attivando così continui processi di controllo e autoregolazione che coinvolgono monitoraggio, valutazione e correzioni (revisioni/ritocchi), in altre parole abilità metacognitive. Schoenfeld esplicita quanto queste competenze possono essere insegnabili.
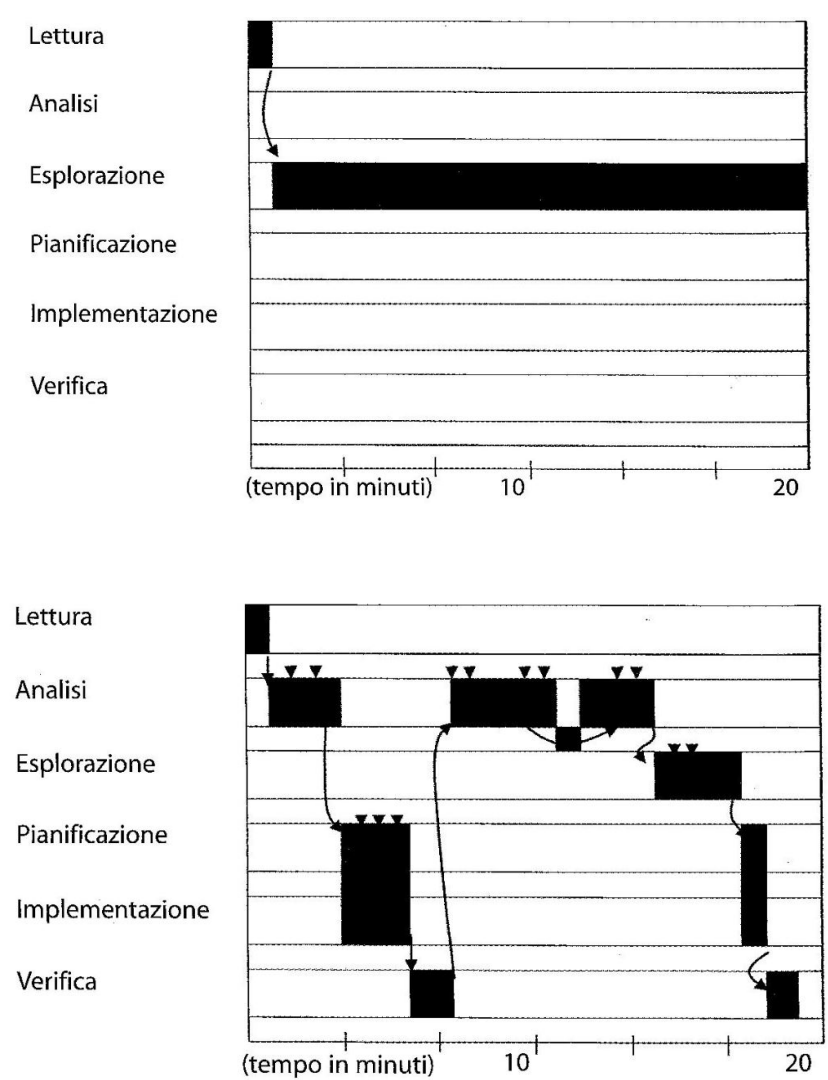

In particolare, Schoenfeld sottolinea come la differenza principale tra il buon solutore e il cattivo solutore è relativa alle domande che il soggetto si pone durante la risoluzione, domande che sono il frutto di un'efficiente attività metacognitiva, e cioè della capacità di riflettere su ciò che si sta facendo e su come lo si sta facendo.

$\mathrm{Nel}$ merito specifico della lettura del testo - oggetto di questo articolo - come sostiene Giasson (2003), la metacognizione è un aspetto decisivo: spesso, infatti, un lettore la cui comprensione del testo è debole presenta anche una metacognizione debole, cioè una scarsa capacità di valutare con obiettività la qualità della propria attività di lettura e comprensione del testo. II ruolo dei fattori metacognitivi nella risoluzione dei problemi è riconosciuto ormai da più di trent'anni. Fin dal 1987, infatti, Lester evidenzia cinque grandi categorie interdipendenti di parametri che influiscono 
sull'attività di risoluzione di problemi:

- conoscenza (comprende i "mezzi" a disposizione quali definizioni, algoritmi, euristiche ecc., ma anche il modo in cui un soggetto organizza, rappresenta e quindi utilizza la propria conoscenza);

- controllo (comprende decisioni esecutive riguardo alla pianificazione, alla valutazione e alla regolazione della conoscenza);

- fattori affettivi;

- convinzioni (il modo in cui un soggetto vede la matematica);

- condizioni socioculturali.

I fattori metacognitivi sono in genere quelli relativi al controllo, alla rappresentazione e alla regolazione della conoscenza, e alle convinzioni; i fattori affettivi, invece, comprendono emozioni e atteggiamenti, fra i quali assumono particolare importanza le motivazioni e gli interessi. Un insieme analogo di elementi personali gioca un ruolo cruciale nell'approccio alla letto-scrittura e nella costruzione stessa del concetto di "lettura", su cui le concezioni evolvono durante il percorso di crescita e di scolarità (Ferreiro \& Teberosky, 1982).

A livello procedurale, in analogia con quanto anticipato da Polya e Schoenfeld, anche in Lesh (2006) e in Sriraman e Lesh (2006) viene evidenziato come l'attività di risoluzione dei problemi includa un certo numero di cicli iterativi e di passaggi da una fase all'altra, nei quali gli allievi si muovono per arrivare alla soluzione, tornando indietro per riflettere sulle ipotesi formulate, affinando ciò che hanno individuato, procedendo attraverso diverse strategie. Non si tratta dunque di un approccio lineare che dai dati porta direttamente al risultato, bensì di un percorso circolare, riconducibile a quello illustrato sulla matematizzazione, e in ciò assume un ruolo fondamentale la lettura del testo e quindi l'importanza di sviluppare negli allievi competenze di tipo trasversale. Risulta ad esempio molto interessante analizzare i processi decisionali che portano un allievo ad adottare una strategia piuttosto che un'altra nella risoluzione di un problema.

I comportamenti fallimentari nella risoluzione di problemi non dipendono quindi solo dalla carenza di conoscenze: la poca efficienza rispetto ai processi di controllo attivati o la loro mancata attivazione influiscono in modo determinante sull'efficacia dell'azione del solutore. In maniera analoga, la mancata consapevolezza delle risorse di cui si dispone comporta l'incapacità di valutare in modo attendibile diversi aspetti, tra i quali il tempo necessario per svolgere un certo compito. Appare dunque importante che l'insegnante sia consapevole di ciò e che presti attenzione a questi aspetti, ovvero che curi, oltre alle risorse cognitive, anche la loro gestione nei processi decisionali. Tutto ciò incentiverebbe il recupero e il potenziamento delle competenze degli allievi nella risoluzione di problemi.

\subsection{La "gerarchia degli errori" di Newman e Clements}

Nell'ottica proposta ricordiamo anche lo studio condotto da Clements (1980), sempre negli anni '80, basato su una "gerarchia degli errori" detta "di Newman e Clements", secondo la quale l'errore può manifestarsi in una delle seguenti fasi:

1) Lettura 

2) Comprensione
3) Trasformazione del testo in un modello matematico
4) Applicazione di procedure aritmetiche o altro
5) Codificazione della risposta

In questa proposta, la lettura viene separata dalla comprensione, dando alla prima sì un ruolo importante per la risoluzione dei problemi, ma, allo stesso tempo, presentandola come un'azione a sé. Da questo punto di vista, ricerche più recenti hanno invece ampiamente confermato che la lettura è essa stessa parte di un processo interpretativo complesso (p. es. Giasson, 1995; Filippini \& Segreto, 1999; D'Amico \& Devescovi, 2013), che non può essere quindi inteso come disgiunto dall'atto del "comprendere": piuttosto, è un'abilità funzionale e simultanea all'operazione stessa di comprensione. Basti ricordare l'assunto chiave per cui la lettura non coincide solo con la decodifica di segni linguistici (cioè con la riconduzione dei segni grafici ai suoni corrispondenti e con il riconoscimento di parole), ma, come vedremo meglio in seguito, prevede l'attivazione di una serie di schemi mentali e di risposte cognitive fondamentali per la comprensione e quindi, nel nostro caso, per l'adeguata scelta e mobilitazione di concetti matematici.

Al di là della scelta, non per forza condivisibile, di considerare la lettura come separata dalla comprensione, la tesi di Clements è quanto mai interessante e valida: egli sostiene infatti, contrariamente a quanto si è soliti ritenere, che il fallimento dei bambini che non riescono a risolvere problemi avviene nelle prime tre fasi, cioè in quelle precedenti l'applicazione di tecniche matematiche. A proposito di questa tesi, D'Amore sostiene:

«credo che qualsiasi buon insegnante di scuola primaria, anche senza compiere apposite ricerche, ne abbia la convinzione:

- il bambino legge il testo ma non lo capisce;

- lo capisce ma non lo coglie in un tutto unico;

- per mancanza di motivazione, non ha stimoli per una completa volizione;

- per mancanza di immaginazione, non sa tradurre il testo scritto in lingua comune in qualche cosa di matematico (grafico o operazione);

- gli manca l'intuizione nell'immaginare la situazione eventualmente tradotta;

- non sa scegliere il modello matematico idoneo:

- non sa crearsi un modello;

- se ne crea uno non adatto, che quindi non lo aiuta nella soluzione.

In fondo, la parte matematica esplicita, che sarebbe comunque I'ultima a comparire, sembra essere l'ostacolo più remoto: arrivarci è già molto, superarlo è ostico (...)».

(D'Amore, 2014, p. 171)

Questa tesi è stata confermata da molte ricerche in didattica della matematica (Mayer, 1982; De Corte \& Verschaffel, 1985; Laborde, 1995; D'Amore, 2000; 2014; Verschaffel, Greer \& De Corte, 2000; Ferrari, P. L., 2004; 2012; Zan, 2007; 2016; Fornara \& Sbaragli, 2013; D'Amore \& Fandiño Pinilla, 2016), che hanno mostrato come tante difficoltà nella risoluzione dei problemi siano legate a componenti linguistiche (lessicali, sintattiche, testuali) che influenzano la rappresentazione, la comprensione e l'interpretazione della situazione. Tra queste incidono le variabili redazionali e gli 
atteggiamenti che gli studenti hanno elaborato nel tempo nei confronti del testo di un problema, e, in particolare, come ci si approccia alla lettura di un problema. Come si è detto, gli insegnanti sono ben consapevoli di questo stato di cose, e notano facilmente che l'allievo spesso legge il testo, una o anche più volte, ma non lo capisce a fondo, non ne mette adeguatamente in relazione le parti oppure non lo coglie nel suo essere un "tutto unico" dotato di senso globale. Ma perché si realizza questa situazione così di frequente? In che modo (o in quali modi) gli allievi tendono a leggere il testo di un problema? Ovvero, come gestiscono questa "porta d'entrata" per raggiungere, interpretare e mettere in gioco gli aspetti matematici?

\section{Leggere un testo per comprenderlo}

\subsection{Che cos'è un testo e che cosa significa comprenderlo}

Quando la comprensione di un problema deve passare attraverso la lettura silenziosa di un testo - e non attraverso altre forme come, ad esempio, l'oralità - il problema di come gli allievi affrontano la lettura è strettamente collegato a due questioni di più vasta portata: che cos'è un testo e come funziona, in generale, la sua comprensione da parte del lettore. Queste questioni vanno poi riconsiderate alla luce delle peculiarità del genere testuale "problema matematico", rispetto al quale ogni aspetto assume determinate specificità.

Per prima cosa, va sottolineato che leggere un testo (anche, apparentemente, bene), di per sé, non significa automaticamente capirlo a fondo: è importante cioè distinguere tra un lettore fluente nella lettura ad alta voce e un lettore efficiente, cioè un lettore che comprende e agisce opportunamente sul testo, perché non è detto che le due cose coincidano. Come sottolinea Colombo (2002, p. 8), «una buona esecuzione orale, con intonazioni e pause adeguate, può essere indizio di una buona comprensione, ma non sempre lo è: si può anche leggere "bene" (in questo senso) senza capire molto». Infatti, leggere un testo per comprenderlo non significa solo decodificarlo con sicurezza, ma ricostruire il suo significato d'insieme collegando le varie parti portatrici di significato. Non a caso, come dice la parola stessa, il testo (dal latino textus, "tessuto") rimanda all'idea di vari fili intrecciati a formare un tutto unico, che sta insieme: tale è, o meglio dovrebbe essere, qualsiasi testo ben formato, efficace per i propri fini comunicativi.

In particolare, le due proprietà base per l'analisi di un testo sono coerenza e coesio$n$. $^{3}$ La coerenza si ha se le varie parti del testo cooperano a costruire un contenuto informativo d'insieme individuabile e tematicamente legato, ed è una caratteristica cruciale affinché la comprensione si realizzi agevolmente. Ciò perché

«il ricevente di un testo ne presuppone (...) la coerenza adottandola come principio-guida della sua interpretazione: data una particolare sequenza di frasi, provvederà via via a scegliere quell'interpretazione e a integrare quelle informazioni implicite che contribuiscono a definirne la coerenza».

(Ferrari, A., 2010a, p. 219)

3. Per un quadro aggiornato delle proprietà del testo secondo la prospettiva di analisi della linguistica testuale si vedano Ferrari, A. (2014), Palermo (2013) e Andorno (2003). 
Mentre legge, quindi, il lettore orienta la sua comprensione dapprima intuendo, poi costruendo man mano la coerenza del testo. Ciò avviene in un processo percepito come unico, ma che consiste nell'interpretazione delle microstrutture testuali (cioè le parole, le frasi, i numeri, le figure ecc.) da legare, poi, in macrostrutture, al fine di stabilire un senso d'insieme, attivando «una molteplicità di processi che avvengono talvolta in parallelo, talvolta in sequenza» (Kintsch \& Van Dijk, 1978, p. 364, traduzione delle autrici).

La coesione è la manifestazione linguistica dei legami del testo (Ferrari, A., 2010b) e a essa possono cooperare in misura più o meno evidente ed esaustiva tutti i livelli della lingua (morfo-sintattico, lessicale, interpuntivo), anche per quanto riguarda il testo dei problemi matematici. Per fare qualche esempio, sono particolarmente significativi i connettivi (parole o locuzioni che individuano le relazioni tra frasi o parti di testo, come perché, poiché, quindi, di conseguenza ecc.), i deittici (forme linguistiche strettamente ancorate alla situazione espressa dal testo, come avverbi quali là, qua, ora, forme pronominali e tempi verbali) e le riprese anaforiche (vale a dire le espressioni che richiamano qualcosa di già detto sia attraverso riprese pronominali come ne, lo ecc., sia attraverso variazioni lessicali; ad esempio, in un testo una classe può essere chiamata "classe", ma anche "gruppo" o ancora "insieme di studenti", e sta al lettore cogliere la coreferenza, ossia il rinvio a uno stesso elemento). È superfluo rimarcare come la padronanza di simili aspetti, fondamentale per l'interpretazione di qualsiasi testo, sia determinante per comprendere il senso esatto di un problema matematico (Orsolini, Fanari \& Maronato, 2005).

Sia sul piano del contenuto, sia su quello della forma, i "fili" del testo che permettono a chi legge di costruirne il significato sono però solo in parte esplicitamente veicolati dalle parole scritte. Infatti, per esigenze di economia della comunicazione (Levinson, 2000), la lingua non dice sempre tutto: molti fili restano nascosti, e sta a una raffinata e allenata abilità cognitiva del lettore ricostruire la trama completa. Prendiamo un semplicissimo esempio come "Ha corso, ma il treno era già partito": per ricostruire una (sensata) interpretazione possibile, ciò che c'è scritto non basta; dobbiamo immaginarci un contesto noto che permetta di stabilire il soggetto (o referente) del testo, dobbiamo comprendere la relazione di significato fra gli elementi citati (che potrebbe essere così riformulata: "sebbene abbia corso, non è arrivato in tempo per prendere il treno"), dobbiamo inferire che il referente "quindi ha perso il treno", e, non da ultimo, dobbiamo possedere sufficienti conoscenze su "come va il mondo" per sapere che spesso per prendere il treno si corre.

Insomma, come scriveva Umberto Eco (1994, p. 34), il testo è generalmente una «macchina pigra», su cui, affinché funzioni, il lettore deve intervenire aggiungendo conoscenze, colmando lacune e producendo inferenze. II problema matematico non si sottrae quasi mai a questa caratterizzazione - quando ha un contesto non esclusivamente matematico - e ciò porta a una conseguenza: per questa tipologia di testi, il problema matematico non è mai un problema solo matematico.

Va considerato che nella scuola elementare in continuità con la scuola media si privilegiano problemi a contesto non solo matematico nella convinzione che possano favorire l'interpretazione delle situazioni legate al reale o che possano aiutare l'allievo. Un'accorta riflessione sugli aspetti linguistici mette però in evidenza che non sempre questa tipologia di testi è di aiuto agli studenti: ciò dipende da come il problema è formulato, in quanto la lingua può sì aiutare, ma anche portare nuovi elementi di ambiguità e potenziale difficoltà (o quantomeno di riflessione). 


\subsection{Il genere testuale "problema matematico"}

II problema matematico classico è un testo particolare, tipologicamente ibrido, che rappresenta per alcuni un vero e proprio "genere letterario" (Gerofsky, 1996; Zan, 2007; 2016). Solitamente si tratta di testi misti, che non possono prescindere dall'interazione tra informazioni rappresentate secondo diversi registri semiotici (aritmetico, algebrico, figurale ecc.), fra le quali l'occhio del lettore deve muoversi per metterle in relazione; inoltre, sono testi che possono includere porzioni di tipo non continuo (cioè forme testuali come grafici e tabelle). A livello di contenuto, per la comprensione di questi testi è spesso richiesto al lettore-solutore di attingere sia dal magazzino delle sue conoscenze disciplinari (semantiche e procedurali), sia da quello delle sue conoscenze generali (cose e situazioni della realtà). A livello pragmatico, va poi considerato che questo genere testuale è di solito eteroposto, in quanto chi deve risolvere il problema (l'allievo) è una persona diversa da chi lo propone (l'insegnante), e questo incide sulla possibile difficoltà dell'allievo a capire il senso della domanda posta (ad esempio se non riconosce la situazione problematica descritta).

Tale genere testuale può realizzarsi in diversi sottogeneri. In particolare, si possono avere problemi assimilabili a meri elenchi di dati e problemi di tipo più narrativo, in cui i dati sono veicolati attraverso una trama testuale che vorrebbe essere maggiormente articolata, caratterizzata dalla presenza di personaggi che compiono azioni mosse da scopi; oltre a questi due sottogeneri, altri problemi si trovano a metà strada tra l'elencativo e il narrativo: questi rappresentano la tipologia più ricorrente tra le proposte scolastiche. Occorre considerare che modelli di problema diversi portano a processi risolutivi diversi.

Greer, Verschaffel e De Corte (2002) definiscono il genere testuale "problema matematico" nel seguente modo:

«un testo (tipicamente contenente informazioni quantitative) che descrive una situazione ritenuta familiare a chi legge, e che pone una domanda quantitativa, la cui risposta può essere ottenuta eseguendo operazioni matematiche sui dati forniti dal testo, o ricavati in altro modo».

(Greer, Verschaffel \& De Corte, 2002, p. 271)

Si tratta di un genere stabilmente presente nell'immaginario scolastico di tutti, che mostra spesso caratteristiche cristallizzate. In particolare, nei problemi sono solitamente riconoscibili questi elementi: una situazione familiare sintetica, che spesso fa riferimento al vissuto extrascolastico, scelta per contestualizzare la struttura matematica su cui si vuol far lavorare gli allievi (che di solito però non è veramente problematica), alcune informazioni quantitative e una o più domande. Queste risultano però spesso artificiose, poiché sono legate quasi esclusivamente ai processi risolutivi più che agli aspetti narrativi della situazione proposta, e che richiedono di solito I'uso di più dati numerici per essere risolte (Zan, 2016). In pratica, I'attenzione dell'autore del testo è di solito rivolta alla struttura matematica, e in particolare alla coerenza logica fra i vari dati forniti dal testo, e non alla struttura narrativa del problema: gli aspetti matematici e quelli narrativi risultano così generalmente poco legati tra loro, cosa che può far perdere di vista la richiesta del problema e provocare una "sospensione di senso" per gli allievi (Schoenfeld, 1991); tale sospensione crea una significativa frattura tra il modo di affrontare i problemi reali e quello in cui si affrontano i problemi scolastici. In particolare, la mancanza o la debolezza di un collegamento 
intrinseco tra contesto e domanda fa sì che la comprensione del testo, nel caso di tali problemi, sia particolarmente focalizzata sulla sola comprensione della doman$\mathrm{da}$, senza che l'allievo si possa appoggiare al "senso" della situazione descritta; di solito, infatti, manca il legame temporale e di causalità che caratterizza le azioni, e gli scopi che le motivano, cioè manca un autentico collegamento con la storia nel suo complesso (Zan, 1998).

Nello specifico, le interpretazioni dei ricercatori portano l'attenzione sul fatto che i testi dei problemi scolastici presentano solitamente alcuni stereotipi, legati sia alla formulazione linguistico-testuale, sia alla struttura matematica: come anticipato, struttura narrativa che non sostiene adeguatamente la domanda, ma che funge da semplice contenitore di dati (evocando, al più, un contesto); presenza esatta di tutte e sole le informazioni e i dati necessari per risolverlo; presenza di una sola soluzione; parole scelte in modo da aiutare intuitivamente a individuare il procedimento risolutivo, senza stimolare una lettura critica del testo e della situazione; dati numerici presenti, così come quelli dei risultati, studiati appositamente per essere dei "bei dati", ossia per essere nella maggioranza dei casi numeri interi; poca coerenza del contesto e debole coesione del testo con la struttura matematica in gioco; esclusivo utilizzo di conoscenze scolastiche acquisite precedentemente; ecc. (si veda ad esempio Verschaffel, Greer, \& De Corte, 2000; Zan, 2007). Questi aspetti non esauriscono tuttavia la problematica; viene infatti anche messa in evidenza la responsabilità delle modalità con cui viene gestita in classe l'attività di risoluzione di problemi, cioè delle norme più o meno implicite che la governano: dare poco tempo a disposizione per risolverlo; applicare solo conoscenze o abilità acquisite da poco tempo; dare poca importanza alla lettura nella comprensione del testo; non favorire processi di controllo ecc.

In sostanza, il processo di lettura e comprensione dei testi è fortemente condizionato sia dall'abitudine a certe caratteristiche testuali ricorrenti, sia dalle esperienze iterate che con determinati testi si fanno. Pertanto, i testi tipici dei problemi matematici e le prassi stereotipe con cui si affrontano favoriscono comportamenti irrazionali negli allievi nell'approcciarsi alla risoluzione, che consistono nel combinare in modo casuale testo, dati e scorciatoie cognitive che hanno interiorizzato nella loro esperienza scolastica precedente. Ciò fa allontanare gli studenti da quell'atteggiamento attivo, critico e costruttivo che favorirebbe lo sviluppo di processi di pensiero significativi, i quali a loro volta consentirebbero di saper prendere decisioni qualitative in base al contesto, oltre che quantitative.

\subsection{Leggere e comprendere un problema matematico: un'attività strategica}

Appurate la natura e le caratteristiche dei testi dei problemi, è evidente che la loro comprensione non può scaturire da una lettura superficiale: questa, al più, come spesso accade, porta a una rappresentazione semantica approssimativa e alla combinazione acritica dei dati matematici. Lavorare per migliorare la comprensione del testo significa offrire agli allievi uno strumento efficace in prospettiva transdisciplinare, e in particolare proprio per quanto riguarda la matematica (lo conferma ad esempio lo studio di Glenberg, Willford, Gibson, Goldberg \& Zhu, 2011).

Includere questo aspetto nella didattica significa anzitutto prendere atto della sua complessità. Infatti, anche per leggere e comprendere un problema matematico vale ciò che è illustrato da molti studi (come De Mauro, 1994; Levorato, 2000; Zanetti \& Miazza, 2004; Lumbelli, 2009; D'Amico \& Devescovi, 2013; specifico sui problemi, 
Zan, 2016), e cioè che "leggere per comprendere" è un processo complesso e multicomponente, cioè un processo che prevede il ricorso, più o meno automatico, a una serie di risorse cognitive e di processi cognitivi (D'Amico \& Devescovi, 2013). Tali componenti possono essere così sintetizzate:

\section{Risorse cognitive}

- memoria, in particolare quella di lavoro (elabora piccole parti di informazione letta e le trattiene per collegarle alle altre, in modo da costruire una rappresentazione sensata del significato);

- conoscenze legate alle forme testuali (cioè alle "grammatiche" dei testi, alle loro strutture: si può pensare ad esempio a quella di un racconto che parla di matematica, ma anche a quelle dei problemi tradizionali);

- competenze e conoscenze linguistiche generali e specialistiche (grammaticali, lessicali, stilistiche ecc.);

- conoscenze concettuali (nel caso specifico quelle matematiche);

- conoscenze enciclopediche (conoscenze generali derivanti dal vissuto o dallo studio, ad esempio che cos'è qualcosa cui il testo fa riferimento);

- conoscenze relative agli script (o sceneggiature comuni rispetto a comportamenti in situazioni specifiche caratterizzate da condotte ricorrenti, su cui Schank, 1982).

Processi cognitivi

- inibizione di informazioni irrilevanti o non pertinenti e riconoscimento di quelle rilevanti;

- formulazione di ipotesi, anticipazioni, presupposizioni e inferenze;

- gestione e, se possibile, integrazione degli impliciti e dei non detti;

- metacomprensione (forma di metacognizione preposta al monitoraggio della comprensione durante e dopo la lettura).

Inoltre, per quanto riguarda più nello specifico la gestione degli aspetti testuali, è ancora esaustivo l'elenco delle competenze necessarie stilato alcuni decenni fa da Bertocchi (1983, cit. in Colombo, 2002):

- competenza tecnica: riconoscere il rapporto tra segni e suoni (cioè saper decodificare il codice alfabetico e le sue peculiarità ortografiche);

- competenza semantica: attribuire significati pertinenti alle parole nel contesto e nel cotesto;

- competenza sintattica: cogliere i rapporti degli elementi costitutivi delle frasi e dei periodi;

- competenza testuale: individuare il tipo di testo, formulare inferenze, ipotesi e anticipazioni;

- competenza pragmatico-comunicativa: ricostruire l'intenzione comunicativa delI'emittente e gli elementi di contesto;

- competenza rielaborativa e valutativa: utilizzare il testo per uno scopo, ponendosi domande su di esso e rielaborandolo in modo opportuno.

Tenere conto delle risorse e dei processi prima elencati, e delle competenze che occorre mobilitare, significa considerare I'atto di leggere un problema matematico nella sua reale complessità. Spesso, invece, nella quotidianità scolastica, la lettura 
del problema è avvertita come un'ovvia condizione di partenza per poterlo, poi, risolvere; un passaggio che si dà per scontato o di cui ci si limita a individuare, genericamente, l'inefficacia. Al contrario, la lettura andrebbe sempre intesa e proposta come intimamente funzionale al successo o all'insuccesso delle azioni che si dovranno compiere su un testo (ad esempio eseguire, applicare, riassumere, validare, interpretare, ricordare, o, nel nostro caso, risolvere ecc.), come mostra, in relazione alla matematica, lo studio di Barton e Heidema (2002).

Questo stesso studio sottolinea che la lettura di testi matematici è una lettura che necessita di concentrazione e di attenzione. Non a caso, solitamente l'atto di leggere un problema è un'operazione silenziosa, per la quale non è richiesta una prestazione ad alta voce (meno adatta a una riflessione profonda tipicamente individuale): come sostiene un allievo di quarta elementare in una ricerca condotta da Zan (1998, p. 45), «Per me un problema e un testo che si deve fare da soli». ${ }^{4} \|$ punto è che di fronte a questo "fare da soli" e in silenzio, gli allievi spesso si trovano privi di strategie e di mezzi, che, invece, andrebbero costruiti e affinati. Insomma, leggere per comprendere non è un mero processo meccanico, ma "un'attività strategica» (Chauveau, 2000, p. 109), cioè «una condotta che coordina più operazioni o più "strumenti" in vista di uno scopo» e che impegna cognitivamente il lettore in modo articolato e complesso: è a questa attività che vanno allenati gli allievi.

In matematica, se lo scopo è arrivare correttamente all' "interpretazione della situazione", è essenziale stimolare nei lettori l'aspetto interattivo (o dialettico) della lettura, che consiste nel riuscire a mettere efficacemente in relazione aspetti linguistici, altre forme di rappresentazione presenti nel testo (grafici, figure, schemi, formule ecc.) e aspetti specifici in senso matematico stretto (concetti, relazioni logiche, dati, procedimenti risolutivi ecc). Tutto ciò, nel contesto della realtà extra-linguistica evocata dal problema. Questi aspetti peculiari della lettura per comprendere - che risulta essere intensiva e analitica - permettono di interpretare più a fondo l'episodio dell'analisi proposto da Schoenfeld (par. 3.3), basato sul porsi domande. Al di là di una prima lettura, è infatti proprio attraverso l'analisi che la lettura diventa, per il solutore, oggetto da indagare e approfondire attraverso interrogativi sul testo nella sua globalità; non a caso, nella Figura 3, relativa al bravo solutore, la lettura e l'analisi ricoprono la maggior parte del tempo dedicato alla risoluzione del problema, mentre nella Figura 2, relativa al cattivo solutore, la fase di analisi viene addirittura saltata.

Qui si celano diversi aspetti da considerare: in particolare, lo scopo dell'allievo di fronte a un testo di un problema è davvero quello di "interpretare la situazione" per il fine di doverlo risolvere? Quali modelli concettuali e quali schemi interpretativi di problema matematico possiedono gli allievi? Le ricerche in didattica della matematica hanno messo in evidenza le convinzioni degli allievi nei confronti del problema matematico (Zan, 1998; 2007), che influiscono sul modo di approcciare questo genere testuale.

Le convinzioni degli allievi inerenti lo scopo del problema matematico sono solitamente rivolte all'atto del risolvere o al mettere in campo conoscenze e abilità, più che alla comprensione della situazione; quest'ultima è di solito lasciata implicita o addirittura omessa nelle considerazioni degli studenti. «Per me un problema è una cosa che bisogna saper risolvere. Un problema è un esercizio-prova per vedere se

4. Qui e in seguito, le formulazioni linguistiche sono quelle originali degli allievi (errori inclusi), così come riportati negli studi di Rosetta Zan. 
una persona ha afferrato l'argomento» (III el.); «ll problema per me è un quiz che serve per vedere che, sea capito quello che li anno insegnato» ( $V$ el.); «ll problema per me è una serie di domande che formano un test, che serve per vedere le capacità di un bambino» (V el.) (Zan, 1998, pp. 41-42). L'attenzione dell'allievo nei confronti del testo risulta quindi direttamente collegata con il processo utilizzare del ciclo della matematizzazione, invece di essere inizialmente rivolta al processo formulare, che prevede la comprensione del testo. Questo tipo di convinzioni, che influenzano gli atteggiamenti e i comportamenti messi in atto durante la risoluzione dei problemi, dipendono da diversi fattori, come ad esempio la tipologia limitata e stereotipata di problemi proposti e le abitudini e le attese del docente, spesso focalizzate sul risultato, piuttosto che sulla situazione o sul processo. Basta riflettere sul fatto che è un'attività didattica assai rara fornire un testo di un problema matematico con il fine di comprenderlo o riformularlo, piuttosto che risolverlo. È significativa, da questo punto di vista, la ricerca condotta da Kilpatrick (1987) con allievi di età compresa tra i 5 e i 12 anni, a cui sono state sottoposte storie senza domande inserite in un gruppo di problemi scolastici; per queste storie la maggior parte degli allievi più grandi ottiene in qualche modo un risultato, pur non essendoci nei testi richieste esplicite in tal senso.

Lo scopo che emerge dalle convinzioni degli allievi è intimamente collegato con ciò che gli allievi pensano essere un problema matematico: "un insieme di dati da mettere insieme con un'operazione"; ciò porta l'allievo a concentrarsi quasi esclusivamente sui dati numerici del testo, sulle parole che aiutano a intuire l'operazione risolutiva e sulla domanda, piuttosto che sulla situazione, dunque sull'intero testo preso come un tutt'uno. Riportiamo qualche frase significativa degli allievi tratta da Zan (1998, pp. 36-37): «Per me un problema è una domanda da risolvere con un'operazione» (IV el.); "Per me il problema è una cosa che seguendo i dati impari le operazioni» (II el.); «Un problema è quando abbiamo del materiale e dobbiamo moltiplicarlo, dividerlo, levarci qualcosa o aggiungerci qualcosa» (III el.).

Le convinzioni e gli atteggiamenti che gli allievi si sono formati nel tempo nei confronti del problema matematico sono coerenti con i fini e con la struttura dei testi stereotipati solitamente proposti. Questi testi sono creati appunto in modo che i dati e la breve storia presente siano abbinati al fine di essere utilizzati per rispondere a una domanda, che consente di applicare particolari conoscenze e abilità. Significativa da questo punto di vista è la seguente convinzione di un allievo di quarta elementare (Zan, 1998, p. 34-35): «Mi fa venire in mente problema di una storietta corta dove finita la storia bisogna risolverla e quando non riesco a concentrarmi sul problema mi immagino sempre: ecco perché l'hanno chiamata problema» (IV el.). È evidente che il genere testuale "problema", con le sue caratteristiche stereotipate prima descritte, è già ben interiorizzato da questo bambino di quarta, ed entra per così dire in conflitto con le strutture di altri testi (nella fattispecie narrativi): I'allievo fa infatti riferimento a una "storietta corta", che però non è una vera storia da capire, ma una sorta di pretesto per chiedergli una risoluzione matematica.

Da queste concezioni sul genere testuale e sulle attese didattiche a esso collegate deriva la messa in campo da parte degli allievi di un "rituale scolastico" (Nesher, 1980) per poter risolvere un problema matematico, che non permette di "penetrare nella situazione problematica" (Boero, 1986): "Nel risolvere un problema scolastico molti bambini sembrano procedere in modo casuale, combinando numeri secondo strategie dettate da inferenze dal testo o da schemi risolutivi interiorizzati nella loro precedente esperienza (...)» (Zan, 1998, p. 27), creando così una frattura tra mondo 
reale e mondo scolastico, essendo quello scolastico un rituale assai distante dalla competenza di problem solving necessaria per gestire e risolvere problemi reali. È in effetti ormai assodato che i bravi e i cattivi solutori di problemi possiedono sistemi di convinzioni diversi e che comportamenti all'apparenza irrazionali risultano comprensibili se interpretati secondo scopi e contesti diversi.

Dal punto di vista didattico occorre quindi lavorare sulle convinzioni e sugli atteggiamenti degli allievi nei confronti del problema matematico: che cos'è, come è fatto, perché viene proposto, come può essere, come lo interpretiamo, come possiamo affrontarlo ecc., fornendo un ampio, variegato, ricco e aperto ventaglio di considerazioni, approcci, tipologie testuali, formati, soluzioni e situazioni che consentano di sviluppare negli allievi competenze legate all'attività di problem solving. In tale percorso occorre dedicare tempo e valore alla comprensione del testo (anzi, dei testi), in particolare alla lettura come porta d'entrata per la comprensione, che rappresenta una fase indispensabile per la risoluzione di un problema.

\subsection{Lettura: una o molte?}

Occorre a questo punto affrontare un altro nodo cruciale quando si parla di lettura come competenza trasversale. Una convinzione comune è che esista un solo tipo di lettura e che, quindi, di fronte al testo di un problema matematico l'approccio sia identico a quello ad altri testi. È invece errato considerare la lettura come un'azione sempre uguale a sé stessa, senza differenze di procedura e di scopo: sarebbe più corretto parlare di letture, cioè di operazioni in parte diversificate a seconda del testo e del suo scopo (cioè di che cosa dobbiamo fare con il testo in questione). Pensiamo a come leggiamo la mappa con le fermate della metropolitana o il tabellone con destinazioni e orari dei treni, e poi pensiamo a come abbiamo affrontato un testo di studio per preparare un esame: ci sarà facile avvertire anche intuitivamente qualche differenza. II contatto con diversi tipi di testo e l'esercizio delle diverse letture e delle strategie a esse associate sono oggi ampiamente sostenuti e promossi sia dalle indagini di dimensione nazionale (come INVALSI, 2018), sia da quelle internazionali. Ciò in particolare al fine di proporre e di testare la lettura per ciò che realmente è, e cioè come «un'attività situata e dotata di senso» (OECD, 2018, p. 13, traduzione delle autrici).

La seguente classificazione dei diversi tipi di lettura - che ne mette in luce le peculiarità a confronto con testi e con scopi diversi - è stata proposta da Tanner e Green (1988), e poi spesso presa come riferimento dagli studi e dalle indagini sul tema:

1) lettura esplorativa o orientativa (skimming): consiste nello scorrere rapidamente e a balzi un testo per scoprire di quale argomento e sotto-argomenti tratta, e per capire se è centrato o no rispetto al proprio scopo di lettura. È una lettura molto utile in vari contesti, in quanto è idonea ad esempio a capire a grandi linee di che cosa parla un opuscolo o un articolo, per valutare se procedere con una lettura più approfondita;

2) lettura selettiva (scanning): consiste nel cercare informazioni e dati specifici in un testo (ad esempio, le parole in un dizionario, I'argomento poligoni in un testo scolastico di matematica, le pagine su Leopardi in un'antologia, ma anche i prez$z i$ in un volantino o un nominativo in un elenco ecc.);

3) lettura estensiva o globale (extensive): ha carattere sequenziale (diversamente dalle precedenti) ed è quella che spontaneamente si impiega nella lettura per 
piacere, ad esempio nella lettura di testi narrativi non troppo impegnativi; si può ricavare un certo tipo di apprendimento, in quanto è una lettura che porta comunque alla sedimentazione di memorie e informazioni (Day \& Bamford, 2002);

4) lettura intensiva o analitica (intensive or narrow): è quella usata per capire a fondo e per interpretare al meglio le richieste del testo, ma anche per studiare e per imparare; prevede perciò che il lettore si soffermi maggiormente sul testo e ne rilegga certi passi. È una lettura in cui chi legge attua regressioni, ipotesi e anticipazioni per cogliere meglio il senso del testo stesso, considera altri elementi come immagini, simboli ecc. (se presenti), integra le informazioni che derivano dai dati testuali e le combina tra loro e a dati extra-testuali, e riflette a fondo sui significati delle parole.

Si può notare che le diverse modalità di lettura sono descritte in relazione ai tipi e ai generi di testo, e agli obiettivi di chi ne fruisce. Uno degli errori più comuni alla base di una lettura inefficace è, infatti, proprio quello di applicare un certo tipo di lettura a un testo che ne richiederebbe un'altra (o più di una): in particolare, leggere solo in modo selettivo testi che, invece, necessiterebbero di una lettura profonda e analitica (intensiva), che tenga conto delle varie parti, le gerarchizzi e le colleghi. Per contro, un lettore competente ed efficace sa adattare il proprio comportamento al testo e a ciò che con esso deve fare.

L'analisi resa possibile dalla lettura intensiva è fondamentale quando si affronta un problema matematico, che, come abbiamo visto, è un genere quanto mai esposto al rischio di una lettura affrettata e superficiale. Per comprendere davvero che cosa un problema esprime e chiede, è invece necessaria una piena comprensione difficilmente realizzabile attraverso la sola lettura esplorativa (utile per farsi un'idea globale) o selettiva (a salti). Eppure, come anticipato (Sowder, 1989; Zan, 2011), la strategia tipica degli allievi di fronte a un testo di matematica, in particolare se si tratta di un problema, è di effettuare proprio una lettura selettiva del testo, finalizzata all'applicazione di strategie alternative: ricerca di dati numerici da combinare per effettuare algoritmi matematici; prova di tutte le operazioni e scelta di quella che dà la risposta più "ragionevole"; ricerca delle "parole chiave" (ad esempio tutti, insieme vuol dire che bisogna sommare, rimaste occorre sottrarre) che suggeriscano il modo di combinare i dati; decisione a priori se la risposta dev'essere maggiore o minore dei numeri dati; ecc., attivando così «una rinuncia a priori a comprendere, in quanto le strategie utilizzate sembrano prescindere dalla comprensione del testo» (Zan, 2011, p. 18). L'allievo tende quindi a non considerare i diversi elementi del testo, specialmente quelli linguistici, ma cerca di inferire il processo risolutivo solo sulla base di particolari elementi caratterizzanti (non di rado enfatizzati nei testi o proposti agli allievi come strategie necessarie per la risoluzione dei problemi: ad esempio sottolineare le parole chiave da associare alle operazioni risolutive), come segnala la letteratura in didattica della matematica.

Questa tendenza tipica della lettura dei problemi trova oggi terreno fertile. Infatti, le più recenti ricerche sull'evoluzione delle prassi di lettura (che avviene sempre più su schermo, spesso muovendosi, e con una concentrazione limitata) sembrano prospettare una generale ascesa della lettura a balzi, veloce, soprattutto nei giovani: «lo skimming (la lettura superficiale) è la nuova normalità nella nostra lettura digitale» (Wolf, 2018, p. 75; su questo anche Ziming, 2005; Rivoltella, 2018), e la cosa non può non avere ricadute su altre occasioni di lettura, che richiederebbero, invece, un approccio ben diverso. Poiché la scuola rimane, oggi, spesso, uno dei pochi ambienti in cui è pos- 
sibile dedicare tempo all'incontro coi diversi tipi e generi testuali, la scoperta e la messa in pratica di diverse strategie di lettura per diversi scopi - tra i quali comprendere e risolvere un problema matematico - va favorita ed esercitata. Ciò per costruire quella competenza di lettura (reading literacy) auspicata dagli standard educativi attuali, che significa saper «comprendere, usare, valutare i testi, saper riflettere su di essi e saper entrare in relazione con essi» (OECD, 2018, p. 11, traduzione delle autrici).

\section{5 "Cattivi" e "bravi" lettori}

Per quanto non sia semplice standardizzare le pratiche di lettura efficaci o poco efficaci (in quanto c'è un'importante componente individuale), la ricerca nel campo della lettura e della comprensione del testo ha una storia lunga, che si è intensificata a partire dalla metà degli anni '70. Ciò perché (secondo Duke \& Pearson, 2002) c'è sempre stata una consonanza di vedute tra ricercatori e insegnanti circa l'opportunità di insegnare a comprendere una lettura (Ferreri, 2002): essa è di fatto una competenza indispensabile e trasversale alle varie discipline (si veda il volume di Colombo \& Pallotti, 2014).

A questa presa di coscienza condivisa, è conseguito il tentativo di provare a individuare e ad affrontare le difficoltà più ricorrenti nei cattivi lettori. Gli studi hanno dimostrato che, comunemente, i cattivi lettori mostrano scarse capacità soprattutto ai seguenti livelli (Ryan, 1980), fondamentali per affrontare anche i problemi matematici: concettualizzare la lettura come ricerca del significato; verificare continuamente la comprensione per accertarsi di stare cogliendo il significato del testo; compiere inferenze e organizzare il contenuto di un brano; rilevare i passaggi più importanti e ordinarli una volta presenti in memoria; completare un testo incompleto, formulare ipotesi, associare appropriatamente un disegno a un messaggio; utilizzare strategie per dare significato al testo o per superare un ostacolo che ha determinato l'interruzione della comprensione; adattare la scelta delle strategie alle varie esigenze che pone la lettura del testo.

Inoltre, i cattivi lettori tendono a una lettura impulsiva e meccanica (ad esempio, omettono di controllare i titoli ed eventuali altre parti legate al testo), non conoscono o non riconoscono le diverse tipologie testuali (cosa che porta ad avere attese inadeguate o a non averne), non gerarchizzano (faticano a selezionare le informazioni: cercano ad esempio di trattenere tutto facendo molta fatica oppure, viceversa, perdono o trascurano elementi essenziali).

Preliminare a tutto ciò è, però, la capacità di scegliere, fra quelli descritti al paragrafo precedente, il tipo o i tipi di lettura adeguati. È quindi fondamentale impostare a scuola un percorso di educazione alla lettura che si proponga di aiutare gli allievi a correggere le abitudini non vantaggiose e a svilupparne di proficue. Un percorso che, a partire dal contatto con molti testi e molte richieste su di essi, miri prima ad aumentare la gamma di modalità di lettura a disposizione, poi a irrobustire la capacità di scegliere quale o quali adottare a seconda del testo e degli scopi. Si tratta, insomma, di arrivare a lavorare anche sulle competenze metastrategiche, cioè sulla capacità di riflettere per «selezionare le strategie più adeguate per lo svolgimento di un compito» (Daloiso, 2013, p. 72); nel nostro caso, la risoluzione di un problema. Questa sensibilità ai tipi di lettura si sviluppa con l'esposizione a testi diversi sui quali interrogarsi. In concreto, esercitare gli allievi a diventare bravi lettori significa lavorare sulle azioni base della lettura enucleate da Chauveau (2000, p. 109), che qui si è provato ad adattare all'ambito matematico: 
- localizzare il tipo di scritto (p. es. È una storia, Ė un elenco di regole di un gioco, $\dot{E}$ un manuale scolastico, $\dot{E}$ un problema ecc.);

- interrogare il contenuto del testo (p. es. Che cosa chiede il testo?, Dove siamo?, Che cosa succede?, Che cosa fa $X$ ?, Che cosa mi fa capire che cos'è successo a $Y$ ?, A che cosa si riferisce questo elemento?, Che informazioni numeriche/geometriche sono presenti nel testo?, Che relazioni numeriche sono presenti nel testo? ecc.), perché farsi domande opportune serve a capire a fondo e ad attivare scenari pertinenti. Come si è accennato al par. 3.1, il ruolo del porsi domande durante la risoluzione di un problema è particolarmente messo in evidenza da Polya tramite la seguente raccomandazione, che rivolge ai suoi lettori e soprattutto agli insegnanti di matematica:

"Quindi un insegnante di matematica ha una grande possibilità. Ovviamente, se egli impiegherà le sue ore di lezione a far eseguire dei calcoli ai suoi studenti, finirà per soffocare il loro interesse, arrestare il loro sviluppo mentale e sciupare l'opportunità che gli si presenta. Invece, se risveglierà la curiosità degli alunni proponendo problemi di difficoltà proporzionate alle conoscenze della scolaresca e li aiuterà a risolvere le questioni proposte con domande opportune, egli saprà ispirare in loro il gusto di un ragionamento originale»;

(Polya, 1945, p. 7)

- esplorare i testi scritti e le loro parti portatrici di senso: singole parole, sequenze di parole da interpretare insieme (come in più di, tanti quanti) e poi frasi o periodi, ma anche più frasi o più periodi in relazione fra loro. La progressione auspicabile si realizza a partire dal soffermarsi sui significati locali per poi metterli in relazione con quelli generali;

- identificare le forme grafiche: per quanto riguarda il codice lingua scritta si intendono lettere, sillabe, parole ecc., da collegare con informazioni rappresentate in modo semiotico diverso (un esempio su tutti è quello dei problemi con informazioni presenti anche in modo figurale/grafico, aritmetico, algebrico ecc.);

- riconoscere alcune parole (quelle che già fanno parte del proprio vocabolario) e individuare quelle di cui non si conosce il significato (sia specialistiche, sia della lingua comune), o di cui non si è certi, o che possono averne più di uno;

- anticipare elementi linguistici o, nel nostro caso, matematici facendo previsioni e ipotesi sul testo, per poi escluderli o confermarli in modo consapevole al procedere della lettura e dell'analisi. Ciò significa attivare campi semantici e concettuali plausibili in modo da ottimizzare la selezione e la ricerca di informazioni, sapendoli, però, escludere qualora, poi, non si rivelassero pertinenti;

- organizzare gli elementi del testo dal punto di vista logico e mettere in relazione le diverse parti: numeri, figure, parole, frasi ecc. (nell'insieme del testo e all'interno delle parti stesse);

- memorizzare (cioè trattenere) le informazioni semantiche per legarle ad altre attraverso la memoria di lavoro (preposta a trattenere e a manipolare l'informazione durante un compito cognitivo) in dialogo con quella a lungo termine; nel caso specifico, si tratta anche di riconoscere e di trattenere elementi e relazioni dal lato matematico. ${ }^{5}$

5. Nei macro-processi di lettura descritti in PISA (OECD, 2018, pp. 12-17), text processing e text management, si possono ritrovare, con altre parole, molte delle azioni qui elencate. 
Queste azioni, lentamente automatizzate dal lettore esperto, dovrebbero rientrare nel bagaglio procedurale del buon lettore. Difficilmente, però, vengono attuate senza esercizio e senza riflessione: non dimentichiamo, infatti, che l'atteggiamento del lettore, soprattutto nei confronti di specifici tipi e generi testuali (ad esempio il problema), migliora sensibilmente solo con l'allenamento e con la motivazione. Valorizzare queste azioni, comprenderne l'utilità e i benefici, farne oggetto di lavoro, e concedere a esse attenzione e tempo adeguati è senz'altro utile per prevenire gli atteggiamenti di lettura negativi più diffusi negli allievi a confronto col problema matematico, e non solo.

\section{Allenare la lettura per comprendere}

Partendo dall'inquadramento teorico qui illustrato è possibile sperimentare in classe percorsi didattici finalizzati a portare gli allievi a concepire il ruolo chiave della lettura per affrontare i vari tipi e generi testuali, e per gestirne i diversi scopi. Nello specifico, il traguardo conclusivo è quello di arrivare a considerare la lettura (intensiva) del testo di un problema matematico come un atto cruciale in quanto finalizzato alla piena comprensione, imprescindibile per passare alla fase risolutiva.

\subsection{Il confronto spontaneo di diversi tipi e generi testuali}

La sperimentazione potrebbe iniziare dalla terza classe di scuola elementare ${ }^{6}$ attraverso il confronto spontaneo (a coppie o a gruppi) di allievi con svariati testi o stralci di testi appartenenti a diversi tipi, generi e anche formati (narrazioni matematiche; filastrocche matematiche; problemi di diverse tipologie, da quella di tipo più elencativo a quella più narrativa; sezioni espositive tratte da manuali scolastici; ma anche testi d'uso quotidiano come volantini, locandine, testi di istruzioni, enciclopedie o dizionari, giornali ecc. ${ }^{7}$, eventualmente anche pagine di siti web), accomunati dalla caratteristica di veicolare contenuti o dati matematici in modo più o meno esplicito e diretto.

\footnotetext{
6. In generale, un percorso di esplorazione dei testi può iniziare anche molto presto: già dalla scuola dell'infanzia, infatti, è utile mettere a confronto i bambini con svariati testi, composti di parole, numeri, figure, da esplorare e scoprire nelle loro caratteristiche e funzionalità (facendo leva sull' «alfabetizzazione emergente», Clay, 1979, cioè sul sapere linguistico-testuale di cui il bambino dispone ancor prima dell'istruzione formalizzata). A seconda della classe, poi, si potranno variare la complessità e la ricchezza degli stimoli proposti, anche rispetto ai traguardi prefissati. In particolare, il percorso qui presentato è adatto dalla terza elementare in poi, quando le abilità di lettura di base (legate alla decodifica) sono generalmente consolidate, e non rappresentano più uno scoglio di per sé: bambini e ragazzi potranno quindi concentrarsi su aspetti più fini dell'interpretazione del testo; non ci sono, invece, limiti "verso l'alto" in quanto un percorso come questo può portare benefici anche a lettori esperti, come gli studenti di scuola superiore.

7. La proposta è in consonanza con le attuali documentazioni ufficiali in vigore nei diversi Paesi: ad esempio, le Indicazioni Nazionali italiane (MIUR, 2012, p. 37) ricordano che «la lettura va costantemente praticata su un'ampia gamma di testi appartenenti ai vari tipi e forme testuali (da testi continui a moduli, orari, grafici, mappe ecc.) per scopi diversi e con strategie funzionali al compito», e il Piano di studio della scuola dell'obbligo ticinese (DECS, 2015, p. 97) raccomanda il ricorso a «tipologie e generi differenti (...), comprese le forme testuali legate alle nuove tecnologie dell'informazione e della comunicazione, come pagine web, ipertesti e grafici».
} 

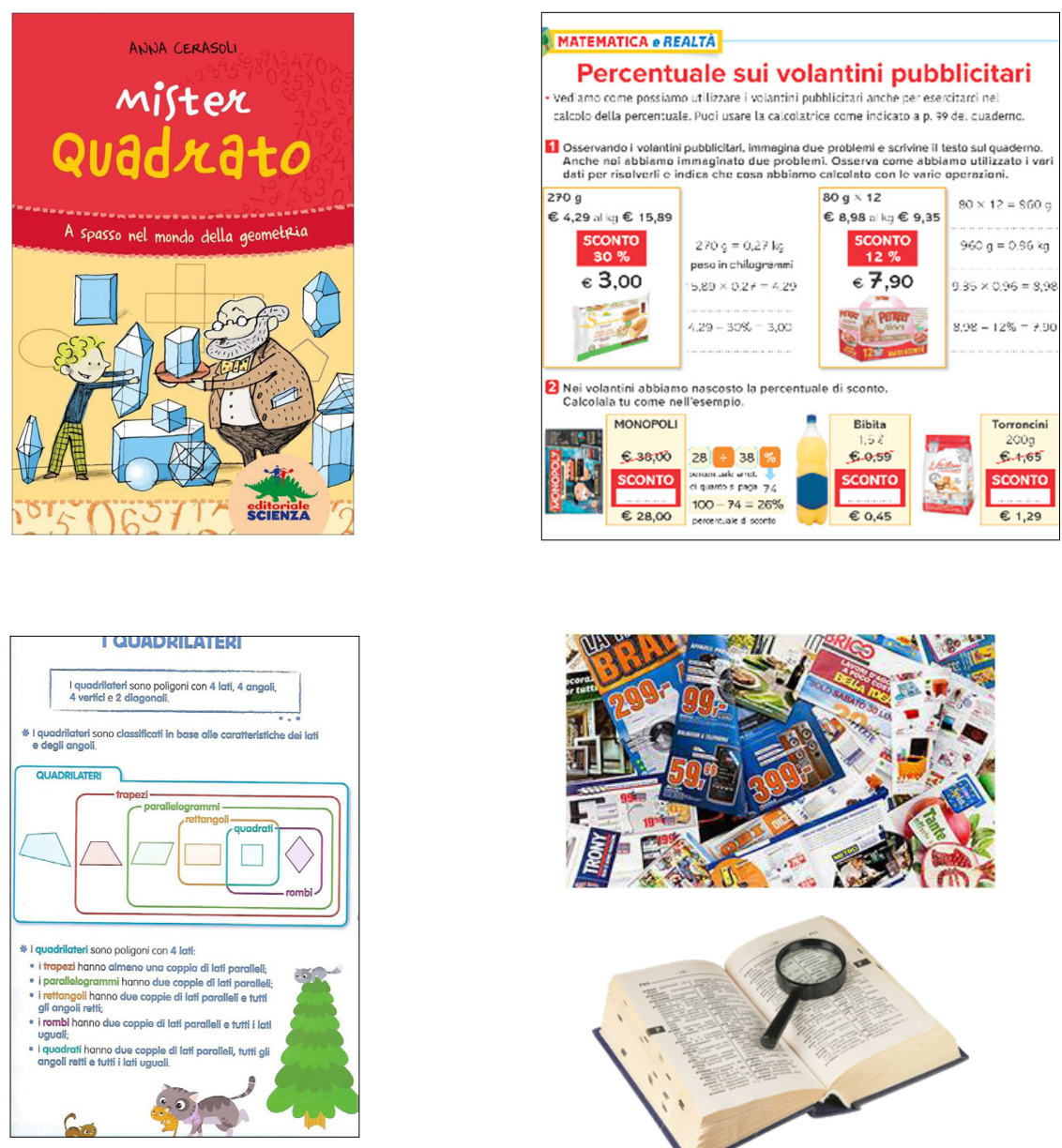

\begin{tabular}{|c|}
\hline Testo della domanda \\
\hline 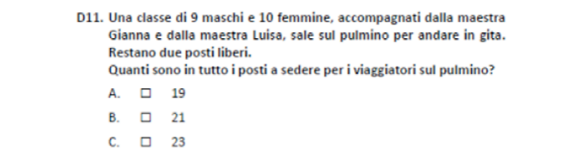 \\
\hline Caratteristiche \\
\hline $\begin{array}{l}\text { SCOPO DELLA DOMANDA } \\
\text { Individuare tutti i dati rilevanti per la soluzione di un problema. } \\
\text { Indicazioni nazionali } \\
\text { Riesce a risolvere facili problemi in tutti gli ambiti di contenuto, mantenendo } \\
\text { il controllo sia sul processo risolutivo, sia sui risultati. }\end{array}$ \\
\hline
\end{tabular}

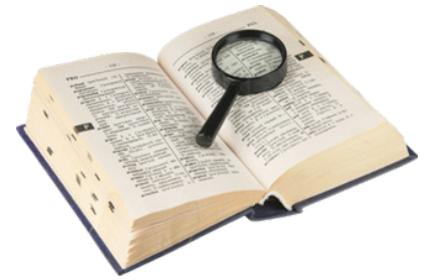

3) UN PROGETTO COMPLESSO

La giovane Sabina si è appena sposata e con il manito ha acquistato un terreno edificabile di forma quadrata che misura $23 \mathrm{~m}$ di lato. Dopo lunghe discussioni, durante un incortro con l'architetto, i due coniugi decidono di voler costruire uma casa con pianta a forma trapezoidale. Inoltre il sogno di Sabina é quello di allevare delle galline per avere uova fresche tutiti i giomi questo del momento che i un'ottma pasticen e adon propar dolc doli d ogri gente Eq questo deve avere forma rettangolare. Davanti alla casa ci deve poi essere un'area piastrellata quadrata sulla quale posizionare un fartastico gill di ulima generazione e un grande tavolo per le cene con gli amici. Infine, i due novelli sposi desiderano avere una piscina dal design innovativo a forma di rombo. Naturalmente deve però anche rimanere dello spazio libero che funga da giardino.

Ė possibile posizionare tutto all' 'ntemo del terreno acquistato? Se si, se tu fossi l'architetto come posizionerestii diversi elementi?
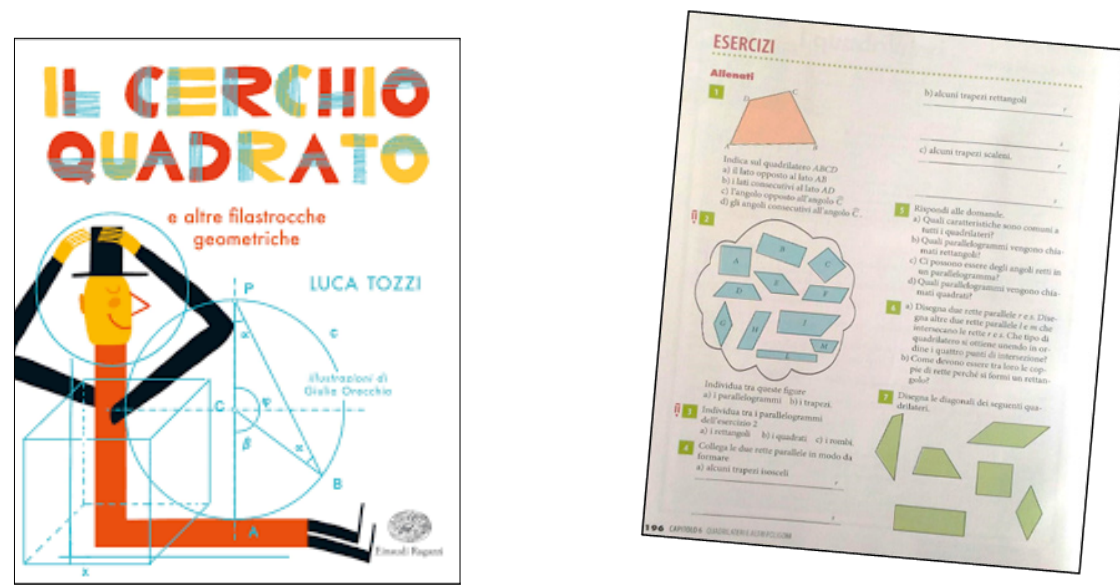
Si chiede agli allievi di leggere i testi proposti, dando loro la possibilità di esplorarli muovendosi liberamente fra essi. Se la classe ha già affrontato una riflessione sul "come" leggere un testo e sui diversi tipi di lettura, nella consegna si può richiamare quanto fatto, chiedendo agli allievi in che modo pensano sia opportuno affrontare i diversi testi. Da questa prima fase, potrà emergere che certi tipi di testi sono meno noti di altri o non lo sono affatto, e andranno scoperti. Per esplorare più in profondità i testi, se ne è stato dato un buon numero, è anche possibile chiedere agli allievi di raggrupparli in base a tratti comuni (lasciando volutamente spontanea e aperta la classificazione): i testi potranno essere raggruppati per tipo, contenuto, lunghezza, formato, difficoltà di lettura, destinatari, presenza o assenza di figure (o di numeri), scopo ipotizzato o definito ecc. Non c'è una sola classificazione corretta, ma molte su cui poi discutere, a partire da somiglianze e differenze: le caratteristiche emerse potranno influire sul fine del testo e, quindi, sulla modalità di lettura. Se la consegna molto aperta dovesse risultare faticosa per studenti poco abituati a riflettere sui testi, si possono prevedere domande-stimolo, finalizzate a innescare la discussione (ad esempio, Quali testi pensate abbiano delle caratteristiche in comune? Quali sono queste caratteristiche? Vi è già capitato di leggerne di simili? Se sì, quando?).

Dopo di che, si può passare a porre ulteriori domande, sempre esplorative, accuratamente formulate per incentivare la riflessione su come è stato affrontato un testo e su che tipo di lettura è stata attuata, raccomandando di fornire motivazioni per le scelte; si tratta di domande-guida che stimolano l'attivazione cognitiva, del tipo Qual è secondo voi lo scopo del testo (o l'intento dell'autore)? Che effetto fa al lettore? Sta chiedendo di fare qualcosa? Sta dando informazioni? Sta raccontando qualcosa? Come avete letto un certo testo? Perché avete fatto questa scelta? Avete letto tutti i testi allo stesso modo? Avete fatto fatica? ecc. Da simili domande, scaturiranno anche le strategie spontanee di lettura, di cui andrà tenuta traccia per poi condividerle al fine di riflettere sulla loro efficacia (o inefficacia) in funzione dello scopo. Eccone alcune di bambini di quarta elementare scaturite da una sperimentazione: "Ho letto a voce un po' più alta ma senza disturbare», "Ho riletto tante volte», "Ho chiesto agli altri che cosa vuole dire questa parola», "Ho cercato di ricordarmi bene quello che avevo letto alla riga prima mentre leggevo la riga dopo perché erano collegate», «Ho segnato le frasi difficili ma che mi sembravano importanti per ricordarmi di andarle a rivedere», «Ho cerchiato le parole importanti, ma alla fine erano troppe», «Ho cercato di capire perché c'erano i numeri», «Ho cercato di capire come collegare tra loro i numeri» ecc. Come si può notare, i bambini, messi in situazione e chiamati a esplicitare quanto fatto, sono in grado non solo di attivare, ma anche di considerare criticamente alcune strategie basilari.

\subsection{Letture diverse a seconda di vari testi e scopi}

Per portare i gruppi a riflettere e a prendere coscienza delle diverse modalità di lettura per testi finalizzati a scopi diversi, è utile prevedere un lavoro specifico su testi identificabili e riconoscibili dal punto di vista del contesto in cui si trovano; ciascun alunno potrà così sperimentare diversi approcci di lettura, soprattutto se il lavoro è integrato da richieste (consegne) alle quali rispondere interagendo col testo: ad esempio, Cercare l'argomento poligoni nel proprio manuale scolastico; Cercare il prezzo più economico dei cellulari presenti in vari volantini; Comprendere un argomento di un libro scolastico; Leggere e saper ripetere oralmente una filastrocca numerica; Leggere una locandina per scoprire quanto costa una settimana di corso per un ragazzino di 
10 anni; Cercare nel dizionario la parola xxx, poi leggere e comprenderne il significato o i significati e in seguito produrre uno o più esempi d'uso della parola in contesto matematico; Dire ai compagni qual è il contenuto matematico della storia; Spiegare con le proprie parole che cosa chiede un problema; Risolvere un problema e così via. I gruppi di lavoro non dovrebbero solo fornire risposte alle richieste, ma dovrebbero anche capire e argomentare come sono arrivati a esse. In concreto, è utile che tengano traccia di come hanno effettuato la lettura, del tempo impiegato per rispondere alla consegna e delle eventuali difficoltà incontrate. Inoltre, riprendendo quanto sostenuto da Polya (par. 3.1) sull'importanza di porsi domande chiave in situazione di problem solving, sarebbe proficuo chiedere agli allievi di annotare che cosa si sono chiesti durante la procedura attuata per arrivare alla risposta. Che cosa devo chiedermi, ad esempio, per trovare l'argomento "poligoni" nel mio testo scolastico? Quali sono le potenziali difficoltà di questa richiesta? Che cosa potrei sbagliare? Che cosa devo sapere? Può non essere sufficiente, infatti, sapere che cos'è un indice e come funziona, in quanto potrebbe non essere banale consultarlo o determinare l'inizio e la fine della trattazione di quell'argomento a livello di contenuto. Se invece la richiesta è di spiegare con parole proprie il testo di un problema, potrei dovermi chiedere, ad esempio, se il sapere matematico in gioco è mantenuto correttamente anche se espresso in forma diversa, o se ho tralasciato parti o, ancora, se i legami fra esse sono rispettati. Anche l'interazione fra pari è un'occasione da sfruttare, poiché saper elaborare insieme richieste adeguate su un testo è indice che di quel testo si sono compresi gli scopi e si ha una buona padronanza; è quindi interessante chiedere agli allievi stessi di proporre consegne per gli altri, di verificarne le risposte, di discuterne le difficoltà. In questa fase, l'insegnante svolge un ruolo chiave: pone a sua volta domande, coglie e valorizza le curiosità, non stigmatizza le risposte non corrette, ma ne fa oggetto di discussione, rilancia i dubbi ascoltando il parere degli altri.

Si noterà come individui diversi, anche all'interno di uno stesso gruppo di lavoro, provino ad attuare strategie di lettura diverse, che si riveleranno più o meno (o per nulla) funzionali allo scopo. Le varie strategie non saranno accolte da tutti come convincenti: proprio per questo si tratta di una fase cruciale, perché attiva la riflessione sullo scopo dei testi e su ciò che il lettore deve fare con essi, ed è essenziale per operare scelte e strategie di lettura consapevoli. In particolare, sarà utile individuare e discutere quegli atteggiamenti di lettura non efficaci, per poter costruire assieme strategie alternative. È in questa fase che può essere utile fissare quanto si è scoperto con un sistema di simboli (di seguito porteremo simboli di animali, spesso usati nelle classi), che evocano le diverse modalità di lettura emerse (qui illustrate al par. 4.4): ad esempio, la lettura di un volantino per capire se contiene delle offerte di giocattoli sarà sicuramente esplorativa, a salti (una lettura che potremmo chiamare "a canguro"); invece, la lettura di una storia matematica, se ben scritta, sarà distesa, estensiva, veloce (come la corsa di un "ghepardo"), ma, a tratti, potrebbe dover essere necessario rallentare e soffermarsi meglio su parole, frasi, numeri o figure. Se poi si leggesse su un manuale scolastico uno specifico argomento matematico, la lettura dovrebbe proprio essere lenta e attenta, intensiva (una lettura a "lumaca", che procede piano e "striscia" fra le parole, i grafici, le notazioni matematiche ecc.). Ma è possibile utilizzare anche analogie e metafore di tipo diverso; in tal senso, durante una sperimentazione in una terza elementare, un'allieva ha associato la lettura di una narrazione matematica a una lettura "a coccole", una lettura calda, confortevole, che rilassa e non richiede troppa fatica, come quella che vive di sera prima di dormire, quando legge un libro sotto le coperte con la sua mamma. 
Figura 5

Immagini di una speri-

mentazione effettuata in

una scuola elementare del

gruppo "Matematica in

rete" (https://rsddm.dm unibo.it/).
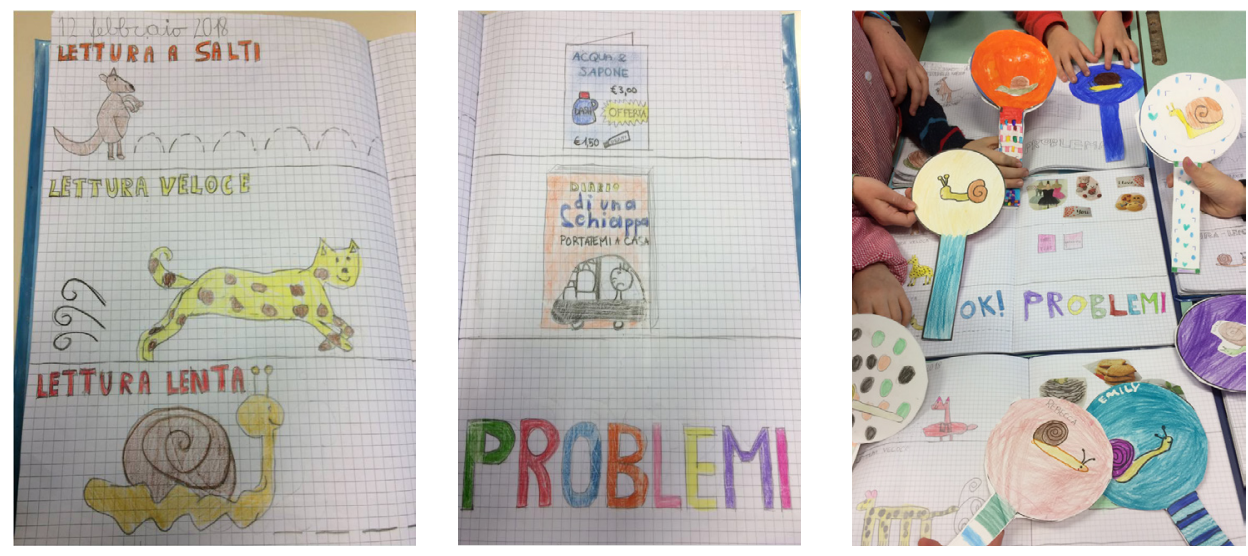

E la lettura per capire e poi risolvere un problema matematico come sarà? Come agiranno spontaneamente gli allievi? Prima andranno veloce, come per leggere una narrazione scorrevole, e poi passeranno a una lettura lenta, per individuare e collegare informazioni utili per risolverlo? O leggeranno subito in modo lento e intensivo? Oppure eseguiranno una lettura a salti? Realizzeranno mappe, disegni o altro? Rileggeranno a voce un po' più alta? Gli atteggiamenti e i gesti saranno diversi, e più o meno efficaci. Occorre a questo punto sottolineare che le ricerche hanno, sì, delineato i principali tipi di lettura (qui illustrati al par. 4.4) in associazione a certi tipi di testo e a certi scopi, ma è fondamentale lasciare agli allievi la possibilità di scoprirli in autonomia (individuando eventualmente tipologie diverse da quelle convenzionali) o di applicare quelli noti con la possibilità di modificarli, provando e sbagliando, per costruire competenza: vanno quindi accettati i loro tentativi, le loro impressioni, ciò che scaturisce dalle esperienze dirette (fallimenti inclusi) e anche la loro terminologia per spiegarsi. Non esistono dunque una via giusta e una sbagliata in assoluto, ma esiste, e vale la pena di proporlo, un lavoro di sensibilizzazione consapevole per gli allievi, che può essere gestito dall'insegnante a seconda del suo stile e dei bisogni della classe.

È insomma significativo inquadrare questa proposta di lavoro nella prospettiva operativa che Trinchero (2012) chiama "ciclo di apprendimento esperienziale», che prevede un momento iniziale di esperienza (nel nostro caso, provare a leggere e a risolvere un problema), seguito da uno di comunicazione (dire che cosa si è fatto), poi dall'analisi di quanto effettuato (confrontare le strategie e i risultati, per cercare di capire quali sono le operazioni convincenti e quali quelle discutibili), dalla generalizzazione (sintetizzare ciò che si è scoperto, cioè come leggere il problema per capirlo a fondo) e dall'applicazione (sperimentare su altri problemi, in altri momenti didattici: cosa che determina il vero apprendimento). Ciò che qui è applicato alla lettura di un problema matematico può ovviamente essere sperimentato su altri generi di testo.

\subsection{Le letture di un problema matematico}

Durante il lavoro basato sul ciclo di apprendimento esperienziale, sarà possibile concentrarsi specificamente sulle diverse letture (o combinazioni di letture) utili per affrontare un problema matematico, partendo dall'intuizione che per questo particolare genere testuale occorre, prima o poi, passare a una lettura attenta e intensiva, finalizzata a comprendere a fondo. Se quest'intuizione non emerge spontanea- 
mente dagli allievi, sarà utile mettere alla prova le altre modalità di lettura emerse; queste funzioneranno solo a volte, mentre in altri casi risulteranno inefficaci per la comprensione e quindi per la risoluzione, soprattutto se il testo del problema non è banale ma presenta qualche scoglio (ad esempio, lessico non noto la cui mancata conoscenza potrebbe compromettere la corretta rappresentazione della situazione; presenza di locuzioni del tipo tanti quanti, non più di, almeno ecc.; dati mancanti; relazioni numeriche complesse; domande non stereotipate ecc.).

In questa fase, è utile che l'insegnante abbia a disposizione e proponga ai gruppi alcuni problemi che presentano possibili difficoltà interpretative come quelle sopra citate. Si dà qui un caso di problema che, come hanno mostrato gli esiti delle prove standardizzate della quinta elementare proposte in Canton Ticino, presentava per i giovani solutori uno scoglio legato al lessico comune (Franchini, Lemmo \& Sbaragli, 2018):

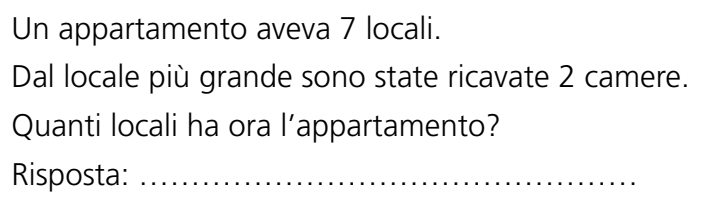

Dai risultati della somministrazione del problema e dalle interviste effettuate successivamente agli allievi emerge che, nella maggior parte dei casi, i bambini non disponevano delle conoscenze lessicali necessarie per comprendere la richiesta matematica. La sinonimia locale-camera, che realizza un richiamo anaforico poco trasparente, così come l'accezione qui pertinente del verbo ricavare non erano parte del magazzino lessicale di molti allievi: sono infatti impieghi lessicali propri del linguaggio specifico legato alla situazione (magari ancora ignota in quanto mai vissuta) della ristrutturazione di una casa. Eppure, la maggior parte dei lettori-solutori ha proceduto alla ricerca di un risultato senza cogliere lo scoglio che sarebbe dovuto emergere in fase di lettura, attribuendo alle diverse parole del testo significati personali ritenuti sensati (per un approfondimento, si veda Franchini, Lemmo \& Sbaragli, 2018, presente nel primo numero di questa stessa rivista). Fornendo agli allievi questo problema in un contesto scolastico diverso dalle prove standardizzate, potrebbe emergere la necessità di lentezza e di riflessione sul testo prima della risoluzione: un buon lettore-solutore, in questo caso, è chi sa fermarsi, capire le difficoltà e prendersi il tempo per provare a superarle (chiedendo i significati dei termini, cercandoli sul vocabolario ecc.). In questo caso, una lettura veloce, a salti, concentrata solo sui numeri, che può essere messa in atto dagli allievi, risulta essere una lettura fallimentare, non efficace in relazione allo scopo del testo. La discussione dei risultati non corretti che possono emergere da una lettura di questo tipo e, a ritroso, la riflessione su ciò che si è fatto per arrivare a essi, porterà l'attenzione all'indietro fino a individuare nella "lettura per comprendere" un passaggio chiave del ciclo della matematizzazione.

Questo semplice esempio ribadisce alcuni aspetti chiave emersi nel corso dell'articolo. Anzitutto, la necessità che le considerazioni sulle modalità di lettura di questo genere testuale siano affiancate a un lavoro quotidiano, critico e costruttivo sul concetto di problema, che contempli una definizione più generale e produttiva possibile di tale concetto, finalizzata a rompere la dicotomia tra problema reale e problema scolastico. Inoltre, occorre che il docente ridefinisca l'obiettivo dell'attività di risoluzione dei problemi in modo da non identificarlo con una prestazione volta a riconoscere le conoscenze e abilità degli allievi, ma come un'esperienza intellettuale stimolante, capace di far sviluppare competenze negli allievi; in questo modo 
si darà un ruolo prioritario alla comprensione del testo nel processo risolutivo e di conseguenza alla sua lettura. Tale ampio obiettivo va poi condiviso con gli studenti e vissuto con loro quotidianamente. Ciò può essere favorito dalla proposta di problemi vari, coinvolgenti, operativi, che rendano l'allievo protagonista della situazione problema e che forniscano occasioni di reale problem solving, ponendo particolare attenzione ai processi, piuttosto che ai prodotti. Problemi, quindi, che portino gli allievi a uscire da certi automatismi improduttivi, a vantaggio di un atteggiamento flessibile e aperto, a partire dalla lettura.

In questa prospettiva l'insegnante - oltre a proporre una pluralità di testi e di attività - può anche stimolare gli studenti a costruire aiuti concreti per la lettura efficace del testo di un problema, così da superare una situazione ricorrente: quella della sola raccomandazione (pur utilissima!) Rileggi, che rischia di risultare sterile. Se l'allievo, pur mosso da buona volontà o da rispetto per le indicazioni dell'insegnante, dovesse rileggere come richiesto, ma senza migliorare l'efficacia della sua lettura, I'operazione potrebbe essere del tutto o in parte infruttuosa, e persino frustrante. Che cosa fare, allora?

Un primo suggerimento è quello di passare da indicazioni generali (Leggi bene, Hai riletto?) a indicazioni operative (Prova a pensare a che cosa fare/a fare alcune cose quando leggi/rileggi), come suggerito anche in Barton e Heidema (2002). Ciò permette anzitutto all'allievo di attribuire rilievo al compito cognitivo della lettura per la risoluzione di un problema, cioè di non orientare la sua attenzione solo alla parte finale del testo, ovvero alla domanda o alle domande, come se fosse slegata dal resto. Per lavorare in questo senso, sarebbe interessante che gli allievi realizzassero una sorta di promemoria (spontaneo e sempre aggiornabile) degli aspetti su cui interrogarsi o delle azioni che potrebbero essere utili da fare prima di passare alla risoluzione di un problema. II promemoria potrebbe contenere simili elementi: controllo se ci sono parole che non conosco o che non mi sono chiare; controllo se ci sono frasi o parti di testo che non capisco bene; segnalo le eventuali relazioni numeriche che non comprendo; segno dove mi sono bloccato e cerco di capire perché; alla fine della lettura, scrivo con parole mie che cosa mi chiede il problema ecc.

Se emergeranno aspetti di criticità, si dovrà lavorare su di essi prima di passare a risolvere il problema (ad esempio cercando i significati delle parole non note, sciogliendo eventuali costrutti sintattici o chiarendo le riprese anaforiche oscure o le relazioni matematiche coinvolte), in modo da rimuovere eventuali ostacoli che potrebbero interferire con la comprensione del contesto e dei processi risolutivi. Consideriamo ad esempio il seguente breve testo di un problema INVALSI del 2013 per il primo ciclo (seconda elementare):

Una classe di 9 maschi e 10 femmine, accompagnati dalla maestra Gianna e dalla maestra Luisa, sale sul pulmino per andare in gita. Restano due posti liberi. Quanti sono in tutto i posti a sedere per i viaggiatori sul pulmino?
a. 19
b. 21
c. 23

Limitandoci agli aspetti linguistico-enciclopedici, quali possibili ostacoli potrebbero interferire con la comprensione del testo, a una lettura veloce? Ad esempio, il significato della locuzione in tutto, ma anche l'anafora viaggiatori, che, tecnicamente, è 
un incapsulatore anaforico, in quanto include in una sola parola la classe di 9 maschi e le 10 femmine, e le due maestre; e poi, siamo certi che per il bambino sia chiaro che l'autista o magari gli autisti (perché grazie alle mie conoscenze enciclopediche so che possono essere più di uno) non siano anche loro viaggiatori? Se per bambini e ragazzi diventa normale prendersi il tempo per leggere e per riflettere sul testo (fermarsi, riformulare con parole loro ecc.), questi aspetti potranno essere affrontati e sciolti prima di passare alle decisioni matematiche.

Oltre a questo lavoro preliminare, una pista operativa per mettere alla prova, con gli allievi, l'efficacia della loro lettura potrebbe essere quella di sfidarli con domande di comprensione non banali. Domande, cioè, che superino quelle spesso circolanti (sovente limitate alla richiesta di reperire informazioni nel testo), ma che richiedano di cogliere il significato di un testo in profondità, collegando opportunamente le informazioni, integrandole o inferendone di ulteriori. Dopo una prima lettura (libera) si potrebbe ad esempio chiedere Di che cosa parla in generale il testo?, Sai dire che cosa ti chiede di fare?, Sai scrivere con parole tue la situazione descritta? Hai già vissuto una situazione simile?, Hai dubbi sul significato di qualche parola, frase o formula?, Hai incontrato informazioni matematiche che non conosci?, Secondo te nel testo manca qualche informazione importante? E domande ancora più puntuali potrebbero essere le seguenti: Chi è il protagonista/chi sono i protagonisti di questo testo? Che cosa deve/devono fare? Che cosa significa la parola matematica XXX o il simbolo YYY?, Quale di queste affermazioni non è corretta? [scelta multipla], Perché il personaggio $X$ ha bisogno di.../dice...?, Che cosa rappresenta questa figura $O$ questo grafico?, Quali relazioni ci sono tra questo testo e questola grafico/figura?, Perché ti viene fornita una certa informazione? È utile per risolvere il problema? Poste dapprima dall'insegnante, le domande pian piano vengono interiorizzate dagli allievi, che arrivano a porsele da soli (secondo la filosofia di Polya) e che, in seguito, possono eventualmente idearne di ulteriori - adeguate a contesti specifici - e rivolgerle ad altri. La formulazione stessa delle domande ci dice qualcosa di come l'allievo sta affrontando il testo, poiché, sostengono gli studi, «una persona capisce qualcosa se è in grado di formulare le domande adeguate rispetto alle cose che deve capire» (Corno, 1990, p. 227; sull'argomento anche Corno, 1992).

Se la lettura è stata superficiale, frettolosa o a salti, alcune di queste domande imporranno necessariamente un ritorno accurato sul testo (cioè una lettura intensiva): sono infatti domande per le quali è difficile reperire la risposta (giusta) solo scorrendo il testo e le sue componenti grafiche, in quanto la formulazione della risposta stessa richiede un'elaborazione profonda dei contenuti. Sarà quindi interessante riflettere insieme sui limiti di efficacia di una lettura esplorativa o selettiva, e su quando, invece, è opportuno rallentare per essere sicuri di capire bene. Al di là di qualche eccezione, si scoprirà attraverso l'esperienza che per capire bene il testo e poi per rispondere alle domande dei problemi la via giusta per sbagliare di meno è procedere con lentezza.

Insomma, il senso dei suggerimenti qui proposti è così sintetizzabile: trasmettere agli studenti il messaggio che sui testi, tutti i testi, anche quelli dei problemi, si può (e si deve ed è utile) discutere e ragionare, arricchendo e migliorando, in questo modo, anche le emozioni e l'immaginario scolastico a essi legato; allenarli a leggere per comprendere, costruendo con loro una gamma di strategie; abituarli a verificare la comprensione prima di procedere con i tentativi di risoluzione, e a rimetterla in discussione anche nel corso del processo risolutivo. In particolare, lungo tutto il per- 
corso e, in seguito, come pratica abituale, è utile dare importanza alle fasi di discussione e verbalizzazione, e incentivarle anche in situazioni didattiche in cui non sono abituali (come, appunto, la discussione rispetto a testi di contenuto matematico): ciò significa, in concreto, discutere in classe sia le caratteristiche e i contenuti dei testi, sia le decisioni prese per gestirli, in modo da esercitare su di esse un controllo metacognitivo. Come sottolinea Chambers (2015), la discussione è un atto fondamentale del Reading Circle, in quanto «alcune discussioni, nella misura in cui ci inducono a riflettere più attentamente e profondamente su quello che abbiamo letto, producono l'effetto di renderci più consapevoli» (Chambers, 2015, p. 18). II messaggio che dovrebbe passare è che anche il problema è un testo con cui si può interagire, sul quale ci si può porre domande e rispetto al quale è possibile essere critici (per la formulazione di domande-stimolo per la riflessione si vedano Chambers, 2006 e Blezza Picherle, 2013).

\section{Il tempo per concludere}

In questo articolo abbiamo mostrato il ruolo inevitabile e decisivo della lettura del testo di un problema matematico allo scopo di risolverlo. Una via possibile per rendere più efficace questa fase potrebbe essere quella di sensibilizzare gli allievi sui diversi tipi di letture dei testi in funzione del contesto, arrivando a definire l'importanza di una lettura intensiva nel caso di problemi matematici, finalizzata alla comprensione. Tale proposta didattica va affiancata a un lavoro ampio e critico sul genere "problema matematico", che consenta di allargarne l'interpretazione da parte di docenti e allievi. In questa prospettiva gioca un ruolo cruciale il tempo, in tutte le sue accezioni e rispetto a tutti gli attori coinvolti: il tempo che il docente si concede per proporre attività ricche e differenziate, per far leggere e interrogare i testi, per ascoltare i dubbi, per porre e rilanciare domande ecc.; il tempo che l'allievo si concede per affrontare un problema, in particolare per comprenderlo e per attivare strategie risolutive; il tempo per una didattica coraggiosa, che sappia dedicarsi a percorsi lunghi. Più in generale, il tempo necessario al lettore per entrare in relazione con un testo complesso, recuperando il gusto per i «pensieri lenti» (Rivoltella, 2018), indispensabili per riflettere. In questo senso, la neuroscienziata Maryanne Wolf (2018) ha sottolineato come la modalità di lettura intensiva sia un'autentica forma di allenamento del cervello a scavare in profondità, rafforzando le sue reti e i suoi processi, in modo che l'analisi e il ragionamento siano modalità operative disponibili anche nella vita di tutti i giorni.

Il tempo non dovrebbe in effetti rappresentare un vincolo per la comprensione di un testo. Ciò è in linea con quanto sostenuto dalla didattica proposta da Emma Castelnuovo basata sul rispetto dei tempi dell'alunno e del suo modo di mettersi alla prova, come spiega questa citazione a lei attribuita:

"Lasciate ai ragazzi il "tempo di perdere tempo", nel senso di garantire loro l'opportunità di costruire soluzioni, anziché far loro usare soluzioni già pronte. Il che è come dire dare loro il tempo per riflettere, per pensare, per ipotizzare, per operare con la mente, per arrivare a capire e, quindi, a costruire conoscenze sicure». 
Se si prende come assunto che la fase di lettura e comprensione di un testo è importante per la risoluzione dei problemi matematici, allora è fondamentale lasciare il tempo agli allievi di viverla e gestirla. II bisogno di tempo per leggere è collegato in prima battuta a necessità fisiologiche, che molti studi recenti hanno permesso di scoprire a fondo (ad esempio Dehaene, 2009; Wolf, 2018): lettura e comprensione di un testo richiedono tempo e concentrazione perché l'atto stesso di leggere ci impegna fisicamente più di quanto avvertiamo. Basti ricordare che, leggendo, l'occhio compie ricorsivamente due tipi di azioni differenti: saccadi (numerosi movimenti per mettere a fuoco le parole nella fovea, la regione centrale della retina) e fissazioni (che durano in media $300 \mathrm{~ms}$ ). Questo solo per ricostruire i segni scritti, cioè le parole, che poi saranno riconosciute e, se note (in quanto presenti nel magazzino lessicale), comprese, mentre, se non sono note, andranno decifrate e scoperte. E si è comunque solo all'inizio del processo di "lettura per comprendere": come si è illustrato al par. 4.3, i processi complessi seguono la fase di decodifica, poiché delle parole va poi individuato, se disponibile, il significato adatto al contesto, e le parole singole vanno poi messe in relazione fra loro e, se presenti, con altri tipi di segni (numeri, figure ecc.) per arrivare a una costruzione di senso soddisfacente. Per quanto un lettore possa diventare esperto, e quindi anche più veloce ed efficiente, leggere per comprendere in profondità è un processo che richiede tempo (lo mostra anche l'esperimento di Duggan \& Payne, 2009), soprattutto quando si tratta di testi complessi come i problemi matematici, che, a livello di rappresentazione di senso, richiedono di pensare per far interagire adeguatamente conoscenze del senso comune e sapere disciplinare. Sul piano didattico in senso lato, il bisogno di tempo è illustrato con efficacia da D'Amore, che, a proposito dell'importanza di lasciare tempo per la risoluzione di un problema matematico, afferma:

«È ben noto a qualsiasi insegnante che c'è un periodo più o meno lungo di latenza, molto differente da soggetto a soggetto; ed è anche ben noto che non sembra esservi una relazione tra durata del tempo di latenza e buona risoluzione del problema: essa sembra dipendere più dallo stile del risolutore, piuttosto che dalla sua minore o maggiore capacità. Tant'è vero che, se si stabiliscono durate di risoluzione fisse, vi sono bambini in difficoltà anche tra i buoni risolutori; anzi l'idea stessa di avere un tempo limitato a disposizione crea blocchi e mette in agitazione. (...) Ci sono bambini che hanno bisogno di questo tempo per vari motivi: scaricare tensione, cercare concentrazione, sentirsi rassicurati da una ricognizione su quel che fanno i compagni, ... In quel periodo, ben noto agli psicologi, si fa la punta alla matita in modo accuratissimo, si mettono in ordine di altezza le matite colorate dell'astuccio, si tolgono le "orecchie" dai quaderni ... (...) Sembra che il tempo di latenza sia necessario al bambino soprattutto per calmare il proprio stato di agitazione, per "estraniarsi" dal lavoro che sta per intraprendere: dunque, psicologicamente parlando, non è tempo buttato via, rientrando invece nel bisogno individuale».

(D'Amore, 2014, pp. 101-102)

Il tempo, dunque, per gli allievi risulta fondamentale per una pluralità di ragioni, sia di tipo psicologico, sia di tipo organizzativo-procedurale. Lo testimoniano le convinzioni sul problema matematico tratte da Zan (1998, p. 44), dove la necessità di avere tempo e di affrontare la risoluzione con calma e tranquillità è citata esplicitamente: «Per me un problema è uno svolgimento di cui bisogna riflettere, pensare. 
Ed è anche una lezione che si svolge nel quaderno di aritmetica, la parola problema mi fa venire in mente una cosa di cui ha bisogno di tempo, è una cosa che bisogna impegnarci capirla. (...)» (IV el.); «Per me un problema è una cosa da risolvere con calma e ragionarci sopra, prima di scrivere nel quaderno. Per capirlo bisogna rileggerlo fino a che non si capisce e se è con più domande farlo un po' alla volta, cioè leggere fino alla prima domanda e farlo, fino alla seconda e farlo e così via» (IV el.). Queste sensazioni e intuizioni dei bambini risultano fondamentali da tenere in considerazione anche alla luce delle ricerche attuali sul ruolo dei fattori affettivi nella risoluzione dei problemi di matematica: lo stato di ansia e di paura derivante anche dalla mancanza di tempo, infatti, incide negativamente sulle prestazioni degli allievi (Zan, 2007). Occorre quindi evitare che la risoluzione di un problema diventi per l'allievo una lotta solitaria contro il tempo, che genera un'alternanza di emozioni negative quali frustrazione, rabbia, ansia, che portano alla rinuncia o incidono profondamente sull'esito finale.

Occorre insomma dare tempo agli allievi di "prendersi il tempo" che ritengono necessario, ma anche provare a insegnare loro a gestirlo nel migliore dei modi durante la risoluzione, in modo che il tempo investito per determinate operazioni non sia "perso", ma "speso bene". Infatti, tra le decisioni strategiche che riguardano la gestione delle risorse durante il processo risolutivo e che indirizzano la direzione che prenderà una soluzione, vi sono anche le decisioni relative alla gestione del tempo (quelle su cui, come abbiamo visto, i cattivi solutori rivelano più difficoltà): quanto tempo dedicare a una fase, quando interrompere una fase per aprirne un'altra, quando chiudere un tentativo risolutivo e aprirne uno nuovo, se è più utile leggere tutti gli esercizi prima di cominciare una verifica o leggerne uno e direttamente risolverlo, valutare la difficoltà o il tempo necessario per ogni domanda, scegliere di dedicarsi solo ad alcuni esercizi ecc. E, prima e durante tutte queste decisioni, c'è la gestione del tempo dedicato alla lettura profonda e all'interrogazione del testo, che è tempo prezioso, su cui si poggia la base per passare con consapevolezza ai passi successivi.

\section{Bibliografia}

Andorno, C. (2003). Linguistica testuale. Un'introduzione. Roma: Carocci.

Barton, M. L., \& Heidema, C. (2002). Teaching Reading in Mathematics. Alexandria, VA: Association for Supervision and Curriculum Development.

Bertocchi, D. (1983). La lettura. Lecce: Milella.

Blezza Picherle, S. (2013). Formare lettori, promuovere la lettura. Riflessioni e itinerari narrativi tra territorio e scuola. Milano: Franco Angeli.

Boero, P. (1986). Sul problema dei problemi aritmetici nella scuola elementare. L'insegnamento della matematica e delle scienze integrate, 9(9), 48-93.

Chambers, A. (2006). Il piacere di leggere e come non ucciderlo. Come imparare a leggere con i ragazzi; traduzione, cura e aggiornamento di M.P. Alignani. Casale Monferrato: Sonda.

Chambers, A. (2015). Il lettore infinito. Educare alla lettura tra ragioni ed emozioni. Ed. italia- 
na a cura di G. Zucchini. Modena: Equilibri.

Chauveau, G. (2000). Come il bambino diviene lettore. Per una psicologia cognitiva e culturale della lettura. Roma: Armando.

Clay, M. M. (1979). Reading: the patterning of complex behaviour. Auckland: Heinemenn.

Clements, M. A. (1980). Analysing children's errors on written mathematical tasks. Educational Studies in Mathematics, 11(1), 1-21.

Colombo, A. (2002). Leggere. Capire e non capire. Bologna: Zanichelli.

Colombo, A., \& Pallotti, D. (Eds.). (2014). L'italiano per capire. Roma: Aracne.

Corno, D. (1990). La comprensione vista da vicino. Italiano \& Oltre, 5, 225-227.

Corno, D. (1992). A domanda risponde. Italiano \& Oltre, 1, 19-21.

Daloiso, M. (2013). Le difficoltà di comprensione del testo scritto in lingua materna e straniera. Un quadro teorico per il recupero della competenza metastrategica. EL.LE. Educazione Linguistica, Language Education, 2(1), 68-87.

D'Amico, S., \& Devescovi, A. (2013). Psicologia dello sviluppo del linguaggio. Bologna: il Mulino.

D'Amore, B. (2000). Lingua, Matematica e Didattica. La matematica e la sua didattica, 1 , 28-47.

D'Amore, B. (2014). I/ problema di matematica nella pratica didattica. Modena: Digital Docet.

D’Amore, B., \& Fandiño Pinilla, M. I. (2016). Una formula per la misurazione oggettiva della difficoltà di comprensione di un testo di matematica da parte degli studenti. Uso valutativo e uso didattico. La matematica e la sua didattica, 1-2, 59-78.

Day, R. R., \& Bamford, J. (2002). Top Ten Principles for Teaching Extensive Reading. Reading in a Foreign Language. 14(2), Disponibile in http://nflrc.hawaii.edu/rfl/October2002/day/ day.html (consultato il 29.03.2019).

De Corte, E., \& Verschaffel, L. (1985). Beginning first graders' initial representation of arithmetic word problems. The Journal of Mathematical Behavior, 4, 3-21.

De Mauro, T. (1994). Capire le parole. Roma-Bari: Laterza.

DECS. (2015). Piano di studio della scuola dell'obbligo ticinese. Disponibile in http://www. pianodistudio.ch (consultato il 29.03.2019).

Dehaene, S. (2009). I neuroni della lettura. Milano: Raffaello Cortina.

Demartini, S., Fornara, S., \& Sbaragli, S. (2017). Numeri e parole. Percorsi di lingua e matematica. Firenze: Giunti scuola.

Demartini, S., Fornara, S., \& Sbaragli, S. (2018). Dalla parola al termine. II cammino verso I'apprendimento del lessico specialistico della matematica nelle definizioni dei bambini. In L. Corrà (A cura di), La lingua di scolarizzazione nell'apprendimento delle discipline non linguistiche (pp. 79-101). Roma: Aracne.

Demartini, S., Fornara, S., \& Sbaragli, S. (in corso di stampa). Se la sintesi diventa un problema. 
Alcune caratteristiche del linguaggio specialistico della matematica in prospettiva didattica. In Atti del XV Congresso Internazionale SILFI “Linguaggi settoriali e specialistici: sincronia, diacronia, traduzione, variazione", Genova, 28-30 maggio 2018. Firenze: Cesati.

Demartini, S., \& Sbaragli, S. (2015a). Geometria e narrazione alla scuola dell'infanzia: un "binomio fantastico". In B. D’Amore \& S. Sbaragli (A cura di), La didattica della matematica, disciplina per l'apprendimento (pp. 67-72). Bologna: Pitagora.

Demartini, S., \& Sbaragli, S. (2015b). Storie di figure, Scuola dell'infanzia, 16(4), 17-18.

Duggan, G. B., \& Payne, S. J. (2009). Text skimming: The process and effectiveness of foraging through text under time pressure. Journal of Experimental Psychology: Applied, 15, 228242.

Duke, N. K., \& Pearson, P. D. (2002). Effective practice for developing reading comprehension. In A.E. Farstrup \& S. J. Samuels (Eds.), What research has to say about reading instruction. Newark, DE: International Reading Association, 205-242.

Eco, U. (1994). Sei passeggiate nei boschi narrativi. Milano: Bompiani.

Ferrari, A. (2010a). "coerenza, procedure di". In R. Simone (A cura di), Enciclopedia dell'italiano Treccani (Vol. 1, pp. 219-222). Roma: Istituto dell'Enciclopedia.

Ferrari, A. (2010b). "coesione, procedure di". In R. Simone (A cura di), Enciclopedia dell'italiano Treccani (Vol. 1, pp. 222-225). Roma: Istituto dell'Enciclopedia.

Ferrari, A. (2014). Linguistica del testo. Principi, fenomeni, strutture. Roma: Carocci.

Ferrari, P. L. (2004). Matematica e linguaggio. Quadro teorico e idee per la didattica. Bologna: Pitagora.

Ferrari, P. L. (2012). Linguaggio, formalismo e costruzione del significato in matematica. In T. Pacelli, C. Coppola \& G. Gerla (A cura di), Logica, linguaggio e didattica della matematica (Vol. 135, pp. 129-141). Milano: Franco Angeli.

Ferreiro, E., \& Teberosky, A. (1982). Literacy before schooling. Ports mouth, NH: Heinemann Educational Books.

Ferreri, S. (A cura di). (2002). Non uno di meno. Strategie didattiche per leggere e comprendere. Firenze: La Nuova Italia.

Filippini, L., \& Segreto, A. (1999). Strategie per leggere. Brescia: La Scuola.

Fornara, S., \& Sbaragli, S. (2013). Italmatica. Riflessioni per un insegnamento/apprendimento combinato di italiano e matematica. In B. D'Amore \& S. Sbaragli (A cura di), La didattica della matematica come chiave di lettura delle situazioni d'aula (pp. 33-38). Bologna: Pitagora.

Fornara, S., \& Sbaragli, S. (2016). Che problema, queste parole! La vita scolastica, 2, 16-18.

Fornara, S., \& Sbaragli, S. (2017). Italmatica. L'importanza del dizionario nella risoluzione di problemi matematici. In F. De Renzo \& M. E. Piemontese (A cura di), Educazione linguistica e apprendimento/insegnamento delle discipline matematico-scientifiche (pp. 211 224). Roma: Aracne.

Franchini, E., Lemmo, A., \& Sbaragli, S. (2017). II ruolo della comprensione del testo nel processo di matematizzazione e modellizzazione. Didattica della matematica. Dalla ricerca 
alle pratiche d'aula, 1, 38-63. Disponibile in http://www.rivistaddm.ch/index.php/2017-01volume/2017-01-franchini-lemmo-sbaragli/ (consultato il 29.03.2019).

Gerofsky, S. (1996). A linguistic and narrative view of word problems in mathematics education. For the learning of mathematics, 16(2), 36-45

Giasson, J. (1995). La lecture: de la théorie à la pratique. Montreal: Gaetan Morin.

Giasson, J. (2003). Metacognizione e comprensione della lettura. In O. Albanese, P. A. Doudin \& D. Martin (A cura di), Metacognizione ed educazione. Processi, apprendimenti, strumenti (pp. 178-188). Milano: Franco Angeli.

Glenberg, A., Willford, J., Gibson, B., Goldberg, A., \& Zhu, X. (2011). Improving Reading to Improve Math. Scientific Studies of Reading, 1-25.

Greer, B., Verschaffel, L., \& De Corte, E. (2002). «The answer in really 4.5»: beliefs about word problems. In G. C. Leder, G. Pehkonen \& G. Törner (Eds.). Beliefs: a hidden variable in mathematics education? (pp. 271-292). Dordrecht: Kluwer Academic Publishers.

INVALSI. (2018). Quadro di riferimento delle prove INVALSI di italiano. Disponibile in https:/l invalsi-areaprove.cineca.it/docs/file/QdR ITALIANO.pdf (consultato il 10.04.2019)

Jupri, A., \& Drijvers, P. H. M. (2016). Student difficulties in mathematizing word problems in algebra. EURASIA Journal of Mathematics, Science and Technology Education, 12(9), 2481-2502.

Kilpatrick, J. (1987). Problem formulating: where do good problems come from? In A. Schoenfeld (Ed.), Cognitive science and mathematics education (pp. 123-147). Hillsdale (NJ): Lawrence Erlbaum Associates.

Kintsch, W., \& Van Dijk, T.A. (1978). Toward a model of text comprehension and production. Psychological Review, 85(5), 363-394.

Kintsch, W. (1988). The use of knowledge in discourse processing: A construction-integration model. Psychological Review, 95, 163-182.

Laborde, C. (1995). Occorre apprendere a leggere e scrivere in matematica? La matematica e la sua didattica, 2, 121-135.

Lesh, R. (2006). New directions for research on mathematical problem solving. In P. Grootenboer, R. Zevenbergen \& M. Chinnappan (Eds.), Identities, cultures and learning spaces (Proceedings of the 29th annual conference of the Mathematics Education Research Group of Australasia, Canberra, Vol. 1, pp. 15-34). Adelaide: MERGA.

Lester, F. (1987). Why is problem solving such a problem? Proceedings PME XI. Montreal.

Levinson, S. C. (2000). Presumptive Meanings. The Theory of Generalized Conversational Implicature. Cambridge (Mass.): The MIT Press.

Levorato, M. C. (2000). Le emozioni della lettura. Bologna: il Mulino.

Lumbelli, L. (2009). La comprensione come problema. Il punto di vista cognitivo. Roma-Bari: Laterza.

Mayer, R. (1982). The psychology of mathematical problem solving. In F. L. Lester \& J. Garofalo (Eds.), Mathematical problem solving. Issues in research (pp. 1-13). Philadelphia: The Franklin Institute Press. 
MIUR. (2012). Indicazioni nazionali per il curricolo della scuola dell'infanzia e del primo ciclo d'istruzione. Disponibile in http://www.pianodistudio.ch/ (consultato il 28.03.2019).

Nesher, P. (1980). The stereotyped nature of school word problems. For the learning of mathematics, I, 41-48.

OECD. (2004). The PISA 2003 Assessment Framework: Mathematics, Reading, Science and Problem Solving Knowledge and Skills. Paris: OECD Publishing.

OECD. (2013). The PISA 2012 Assessment and Analytical Framework: Mathematics, Reading, Science, Problem Solving and Financial Literacy, OEC Paris: OECD Publishing.

OECD. (2016). The PISA 2015 Assessment and Analytical Framework: Science, Reading, Mathematic and Financial Literacy. Paris: OECD Publishing.

OECD. (2018). The PISA 2015 Assessment and Analytical Framework: Science, Reading, Mathematic and Financial Literacy. Paris: OECD Publishing. Disponibile in https://www.oecd.org/ pisa/data/PISA-2018-draft-frameworks.pdf (consultato il 27.03.2019)

Orsolini, M., Fanari, R., \& Maronato, C. (2005). Difficoltà di lettura nei bambini. Roma: Carocci.

Palermo, M. (2013). Linguistica testuale dell'italiano. Bologna: il Mulino.

Polya, G. (1945). How to solve it. Princeton: University Press. [Traduzione italiana: 1967, Milano: Feltrinelli].

Ryan, M. L. (1980). Fiction, Non-Factuals, and the Principle of Minimal Departure. Poetics, 9, 403-422.

Rivoltella, P. C. (2018). Leggere e scrivere in digitale. Cosa cambia per il cervello? Avvenire, 5 ottobre 2018. Disponibile in https://www.avvenire.it/opinioni/pagine/leggere-e-scrivere-in-digitale-cosa-cambia-per-il-cervello (consultato il 28.03.2019).

Sbaragli, S., \& Franchini, E. (2017). Valutazione didattica delle prove standardizzate di matematica di quinta elementare. Locarno: SUPSI, Dipartimento formazione e apprendimento.

Schank, R. C. (1982). Reading and Understanding. Hillsdale, NJ: Erlbaum.

Schoenfeld, A. H. (1983). Episodes and executive decisions in mathematics problem solving. In R. Lesh \& M. Landau (Eds.), Acquisition of mathematics concepts and processes (pp. 345-395). New York: Academic Press.

Schoenfeld, A. H. (1987). What's all the fuss about metacognition? In A. H. Schoenfeld (Ed.), Cognitive Science and Mathematics Education (pp. 189-215). Hillsdale, NJ: Lawrence Erlbaum Associates.

Schoenfeld, A. H. (1991). On mathematics as sense-making: an informal attack on the unfortunate divorce of formal and informal mathematics. In J. F. Voss, D. N. Perkins \& J. W. Segal (Eds.), Informal reasoning and education (pp. 311-343). Hillsdale, NJ: Lawrence Erlbaum Associates.

Schoenfeld, A. H. (1992). Learning to Think Mathematically: Problem Solving, Metacognition, and Sense Making in Mathematics. In A. G. Douglas (Ed.), Handbook of Research on Mathematics Teaching and Learning. A project of the National Council of Teachers of Mathematics. New York: Macmillan Publishing Company. Disponibile in http://hplengr.e ngr.wisc.edu/Math Schoenfeld.pdf (consultato il 16.04.2018). 
Sowder, L. (1989). Searching for affect in the solution of story problems in mathematics. In D. B. McLeod \& V. M. Adams (Eds.), Affect and mathematical problem solving: A new perspective (pp. 104-113). New York: Springer.

Sriraman, B., \& Lesh, R. (2006). Modeling conceptions revisited. Zentralblatt für Didaktik der Mathematik, 38(3), 247-254.

Tanner, R., \& Green, C. (1988). Tasks for teacher education. A reflective approach. Harlow: Addison Wesley Longman.

Trinchero, R. (2012). Costruire, valutare, certificare competenze. Proposte di attività per la scuola. Milano: Franco Angeli.

Verschaffel, L., Greer, B., \& De Corte, E. (2000). Making sense of word problems. Lisse, The Netherlands: Swets \& Zeitlinger.

Wolf, M. (2018). Lettore, vieni a casa. I/ cervello che legge in un mondo digitale. Milano: Vita e Pensiero.

Zan, R. (1998). Problemi e convinzioni. Bologna: Pitagora.

Zan, R. (2007). La comprensione del problema scolastico da parte degli allievi: alcune riflessioni. L'insegnamento della matematica e delle scienze integrate, 30 A-B, 6, 741-762.

Zan, R. (2011). L'errore in matematica: alcune riflessioni. PQM 2010/2011. Disponibile in http://www.liceocapece.gov.it/wp-content/uploads/2016/10/R.Zan-Lerrore-in-matemati ca-alcune-riflessioni.pdf (consultato il 20.08.2017).

Zan, R. (2016). I problemi di matematica. Difficoltà di comprensione e formulazione del testo. Roma: Carocci.

Zanetti, M. A., \& Miazza, D. (2004). La comprensione del testo. Modelli e ricerche in psicologia. Roma: Carocci.

Ziming, L. (2005). Reading behavior in the digital environment: Changes in reading behaviour over the past ten years. Journal of Documentation, 61(6), 700-712.

\author{
Autori/Silvia Demartini e Silvia Sbaragli \\ Dipartimento formazione e apprendimento - SUPSI di Locarno, Svizzera \\ silvia.demartini@supsi.ch, silvia.sbaragli@supsi.ch
}

